\title{
Magnetic excitations in polyoxotungstate-supported
}

\section{lanthanoid single-molecule magnets: an inelastic}

\section{neutron scattering and ab initio study}

Michele Vonci,,$^{\dagger}$ Marcus J. Giansiracusa,,$^{\dagger}$ Willem Van den Heuvel, ${ }^{\dagger}$ Robert W. Gable, ${ }^{\dagger}$ Boujemaa Moubaraki, ${ }^{+}$Keith S. Murray, ${ }^{+}$Dehong Yu, ${ }^{\S}$ Richard A. Mole ${ }^{*}$, Alessandro Soncini ${ }^{*}+{ }^{\dagger}$ Colette Boskovic ${ }^{* \dagger}$

$\dagger$ School of Chemistry, University of Melbourne, Parkville, Victoria, 3010, Australia

$\$$ School of Chemistry, Monash University, Clayton, Victoria, 3800, Australia

$\S$ Australian Nuclear Science and Technology Organisation, Locked Bag 2001, Kirrawee DC New South Wales 2232, Australia 
ABSTRACT. Inelastic neutron scattering (INS) has been used to investigate the crystal field (CF) magnetic excitations of the analogs of the most representative lanthanoid-polyoxometalate single-molecule magnet family: $\mathrm{Na} 9\left[\mathrm{Ln}\left(\mathrm{W}_{5} \mathrm{O}_{18}\right)_{2}\right](\mathrm{Ln}=\mathrm{Nd}, \mathrm{Tb}, \mathrm{Ho}, \mathrm{Er})$. Ab initio Complete Active Space Self Consistent Field/Restricted Active Space State Interaction calculations, extended also to the Dy analog, show good agreement with the experimentally determined low lying CF levels, with accuracy better in most cases than that reported for approaches based only on simultaneous fitting to $\mathrm{CF}$ models of magnetic or spectroscopic data for isostructural $\mathrm{Ln}$ families. In this work we demonstrate the power of a combined spectroscopic and computational approach. Inelastic neutron scattering has provided direct access to CF levels, which together with the magnetometry data, were employed to benchmark the $a b$ initio results. The ab initio determined wavefunctions corresponding to the CF levels were in turn employed to assign the INS transitions allowed by selection rules and interpret the observed relative intensities of the INS peaks. Ultimately we have been able to establish the relationship between the wavefunction composition of the $\mathrm{CF}$ split $\mathrm{Ln}^{\mathrm{III}}$ ground multiplets and the experimentally measured magnetic and spectroscopic properties for the various analogs of the $\mathrm{Na}_{9}\left[\mathrm{Ln}\left(\mathrm{W}_{5} \mathrm{O}_{18}\right)_{2}\right]$ family. 


\section{Introduction}

Single-molecule magnets (SMMs) possess an energy barrier to magnetization reversal that is of molecular origin, ${ }^{1-8}$ with lanthanoid-based SMMs (Ln-SMMs) of interest as they can exhibit very large energy barriers and slow magnetic relaxation at relatively high temperatures. ${ }^{9-12}$ Quantum tunnelling of the magnetization (QTM) is a prominent feature of Ln-SMMs and can be switched on and off by the application of small magnetic fields. This possibility may ultimately provide the superposition of logic states necessary for SMMs to act as qubits in quantum computers. ${ }^{13-16}$ Qubits can serve simultaneously as the processor and memory of the quantum computer, with entanglement giving rise to parallel processing that allow calculations to be performed significantly faster than with conventional computers. Ultimately, quantum computers will be able to perform tasks that are impossible for conventional computers, with likely applications in data security and encryption, data management and searching and, more generally, modelling complex natural and physical systems. ${ }^{17,18}$

The interactions that determine the electronic structure for the $4 \mathrm{f}$ states of $\mathrm{Ln}^{\mathrm{III}}$ ions in Ln-SMMs are, in order of decreasing magnitude, electron-electron repulsion, spin-orbit coupling and crystal field (CF), resulting in an energy spectrum of ${ }^{2 S+1} L_{J}$ multiplets. In a manner that depends on the type and symmetry of the donor atoms, and on the Kramers or non-Kramers nature of the $\mathrm{Ln}^{\mathrm{III}}$ ion, the CF splits the degeneracy of the ground state ${ }^{2 S+1} L_{J}$ multiplet into at most $2 J+1$ microstates. An energy barrier to magnetization reversal is observed in Ln-SMMs when a doubly degenerate (or almost doubly degenerate), high magnetic moment ground microstate is stabilized with a large anisotropy barrier separating the two degenerate (or quasi degenerate) components. The effective energy barrier $U_{\text {eff }}$ to magnetization reversal can be experimentally determined from alternating current magnetic susceptibility measurements, 
assuming an Arrhenius-like law for the temperature dependence of the spin-lattice relaxation time of the system. ${ }^{19}$ This energy barrier correlates with the energy gap between the ground state and an excited CF level, commonly the first but not always, ${ }^{11,20,21}$ level of the $2 J+1$ ground state manifold, through which the relaxation process is assumed to occur via an Orbach mechanism. Mismatches between the magnitude of reported $U_{\text {eff }}$ and CF energies are however common and suggest that other relaxation mechanisms are present, and reliable experimental methods and improved theoretical models are required to clarify the dynamics of relaxation., ${ }^{8,22}$

Well established experimental techniques for investigating the electronic structure of LnSMMs, especially coupled with fitting the data to CF models, are magnetometry, torque magnetometry, EPR, paramagnetic NMR and optical spectroscopies. ${ }^{23-26}$ A potentially very powerful technique for the study of CF excitations in SMMs, is inelastic neutron scattering (INS). This technique has the advantage of providing a direct probe of the energy of excited CF levels that lie within the energy transfer of the incident neutron beam. Widely used for studying SMMs based on transition metals, ${ }^{27}$ INS has been employed relatively little for Ln-SMMs. ${ }^{28-33}$ This is due to both the high neutron absorption cross section of Dy, which is the most prevalent $4 f$ metal in Ln-SMMs, ${ }^{5}$ and the usually large splitting, up to hundreds of wavenumbers (tens of $\mathrm{meV}$ ), of the CF levels in some Ln-SMMs, which renders the CF excitations beyond the kinematic range of many INS spectrometers. In fact, the most detailed INS studies on Ln-SMMs reported to date (van Slageren et al. and Dreiser et al.), are complemented by other experimental techniques such as far-infrared and optical spectroscopies. ${ }^{29,34}$ Both of these studies also demonstrate the potentially fruitful interplay of experimental data, ab initio Complete Active Space Self Consistent Field/Restricted Active Space State Interaction (CASSCF/RASSI) calculations and CF models. 
From simple yet powerful electrostatic methods, ${ }^{3,35}$ to computationally inexpensive CF approaches, ${ }^{24,36-38}$ to more computationally demanding ab initio CASSCF/RASSI methods, ${ }^{39-41}$ there is a vibrant debate regarding the theoretical approaches to rationalizing - or predicting - the magnetic properties of Ln-SMMs. ${ }^{8,42-44}$ Ab initio CASSCF/RASSI methods have an intrinsically higher heuristic value as they aim for an accurate description of the wavefunctions, relying on virtually no empirical data beyond the atomic positions obtained from crystal structure determination. However, they sometimes afford errors larger than $20 \%$ in comparison to experimentally-determined CF energies, for reasons that are yet to be fully clarified. ${ }^{29,43}$ On the other hand, CF approaches based on the fitting of spectroscopic data are useful and timeeffective, especially in the presence of high molecular symmetry, which reduces the number of allowed CF parameters. However, these approaches necessarily involve lengthy experimental characterization processes and suffer from over parameterization when the local symmetry of the lanthanoid site is low, as elucidation of the full CF Hamiltonian involves the simultaneous determination of up to $27 \mathrm{CF}$ parameters. ${ }^{37}$

While the majority of Ln-SMMs are conventional coordination or organometallic complexes with organic ligands, the use of inorganic polyoxometalate (POM) ligands in lanthanoid-polyoxometalate (Ln-POM) complexes provide Ln-SMMs with some different characteristics. ${ }^{45-47}$ An advantage offered by polyoxotungstate ligands is the possibility of accessing nuclear spin free systems, which is beneficial for minimizing quantum decoherence and unwanted relaxation. ${ }^{48,49}$ Another advantage of POM ligands is the ability to magnetically isolate the individual $\mathrm{Ln}^{\mathrm{III}}$ centers from each other via the steric bulk of the diamagnetic POM. The most well-studied family of Ln-POM SMMs is the $\left[\mathrm{Ln}\left(\mathrm{W}_{5} \mathrm{O}_{19}\right)_{2}\right]^{9-}$ family, first synthesized by Peacock and Weakley in 1971 and subsequently studied for luminescence properties. ${ }^{50-52}$ 
More recently Coronado and coworkers have performed detailed studies involving bulk magnetic susceptibility, heat capacity and EPR spectroscopy; finding SMM behavior for the Ho, Er, Gd and $\mathrm{Nd}$ analogs. ${ }^{42,49,53-57}$. In these investigations the electronic structure of the members of the $\left[\mathrm{Ln}\left(\mathrm{W}_{5} \mathrm{O}_{19}\right)_{2}\right]^{9-}$ family were initially determined by simultaneous fitting of the magnetic susceptibility data to a CF Hamiltonian and subsequently improved by calculations utilizing an effective point charge model. Interestingly, the SMM behavior of the Ho analog is maintained following encapsulation within a supramolecular silver-alkynyl cage. ${ }^{58}$

We recently communicated the first $a b$ initio CASSCF/RASSI calculations and INS measurements for a Ln-POM complex carried on the non-SMM Tb analog of the family $\mathrm{Na}_{9}\left[\mathrm{Ln}\left(\mathrm{W}_{5} \mathrm{O}_{18}\right)_{2}\right]^{32}$ This combined experimental and theoretical approach shed new light on the sensitivity of the electronic structure of the $\mathrm{Tb}^{\mathrm{III}}$ ground and excited states to small structural distortions from axial symmetry. Here we extend the INS investigation to the SMM members of the family $(\mathrm{Ln}=\mathrm{Nd}, \mathrm{Ho}, \mathrm{Er})$, showing that this spectroscopic technique can be extremely useful for investigating the CF levels of Ln-SMMs. The study is complemented by ab initio CASSCF/RASSI calculations, also including the Dy analog. This type of calculations are relatively uncommon for POM-based SMMs, ${ }^{32,33,59}$ and with this work we show how they can be reliably used for interpreting and rationalizing the spectroscopic and magnetic properties of the investigated lanthanoid complexes.

\section{Experimental Section}

Synthesis. All chemicals were used as purchased with no further purification. $\mathrm{Na}_{2} \mathrm{WO}_{4} \cdot 2 \mathrm{H}_{2} \mathrm{O}$ (Strem, 99+\%), $\mathrm{Y}\left(\mathrm{NO}_{3}\right)_{3} \cdot 6 \mathrm{H}_{2} \mathrm{O}$ (Strem, 99.9\%), $\mathrm{Nd}\left(\mathrm{NO}_{3}\right)_{3} \cdot 6 \mathrm{H}_{2} \mathrm{O}$ (Aldrich, 
99.9\%), $\mathrm{Tb}\left(\mathrm{NO}_{3}\right)_{3} \cdot 5 \mathrm{H}_{2} \mathrm{O}$ (Aldrich, $99.9 \%$ ), Dy( $\left(\mathrm{NO}_{3}\right)_{3} \cdot 6 \mathrm{H}_{2} \mathrm{O}$ (Strem, 99.9\%), $\mathrm{Ho}\left(\mathrm{NO}_{3}\right)_{3} \cdot 5 \mathrm{H}_{2} \mathrm{O}$

(Sigma-Aldrich, 99.9\%), Er( $\left.\mathrm{NO}_{3}\right)_{3} \cdot 6 \mathrm{H}_{2} \mathrm{O}$ (Aldrich, 99.9\%), glacial acetic acid (Chem-Supply, 99.7\%), deuterium oxide (Sigma-Aldrich, 99.9\%).

$\mathbf{N a} 9\left[\mathbf{Y}\left(\mathbf{W}_{5} \mathbf{O}_{18}\right)_{2}\right] \cdot 35 \mathrm{H}_{2} \mathrm{O}(\mathbf{Y}) \cdot \mathrm{Na}_{2} \mathrm{WO}_{4} \cdot 2 \mathrm{H}_{2} \mathrm{O}(14.84 \mathrm{~g}, 45.00 \mathrm{mmol})$ was dissolved in water $(50 \mathrm{ml})$ upon heating to $85^{\circ} \mathrm{C}$. The $\mathrm{pH}$ was then adjusted to 7.2 with glacial acetic acid, maintaining the tungstate solution at constant temperature. $\mathrm{Y}\left(\mathrm{NO}_{3}\right)_{3} \cdot 6 \mathrm{H}_{2} \mathrm{O}(1.725 \mathrm{~g}, 4.500$ mmol) previously dissolved in hot water $(4 \mathrm{ml})$, was added dropwise to the tungstate solution with vigorous stirring. During the addition a fine precipitate formed. After the addition was completed, the solution was left stirring for a further five minutes. The mixture was then cooled to room temperature and the fine precipitate that appeared during the addition of yttrium nitrate was filtered off. The clear solution obtained was stored at $5{ }^{\circ} \mathrm{C}$ for 24 hours resulting in a colorless microcrystalline product. The colorless crystalline product was recrystallized from hot water and after few days of slow evaporation at room temperature blade shaped flat crystals of $\mathbf{Y}$ were obtained (2.1 g, $0.63 \mathrm{mmol}, 12 \%)$. Selected IR data $\left(\mathrm{KBr}, \mathrm{cm}^{-1}\right)$ : $938(\mathrm{~m}), 844(\mathrm{~s}), 777(\mathrm{~s})$, 710 (s), $544(w), 492(w), 427$ (s), 414 (s). Elemental analysis for $\mathrm{Na}_{9}\left[\mathrm{Y}_{(}\left(\mathrm{W}_{5} \mathrm{O}_{18}\right)_{2}\right] \cdot 35 \mathrm{H}_{2} \mathrm{O}$, $\mathrm{H}_{70} \mathrm{YNa}_{9} \mathrm{O}_{71} \mathrm{~W}_{10}$, calcd (found): H 2.1 (1.9), Y 2.7 (1.8), Na 6.2 (5.9), W 55 (54).

$\mathbf{N a} 9\left[\mathbf{Y}\left(\mathbf{W}_{5} \mathbf{O}_{18}\right)_{2}\right] \cdot \mathbf{3 5 D}_{2} \mathbf{O}\left(\mathbf{Y}^{\mathbf{D}}\right)$. A large quantity of $\mathbf{Y}(3-4 \mathrm{~g})$ was heated at $150{ }^{\circ} \mathrm{C}$ under reduced pressure $(100 \mu \mathrm{m} \mathrm{Hg})$ for $6 \mathrm{~h}$. The resulting amorphous powder was recrystallized from the minimum amount of hot $\mathrm{D}_{2} \mathrm{O}(3-4 \mathrm{ml})$ working under a nitrogen atmosphere. Colorless crystals of $\mathbf{Y}^{\mathbf{D}}$, the unit cell of which matched that of $\mathbf{Y}$, were obtained by crystallization at room temperature almost quantitatively. Crystals were quickly filtered, dried under nitrogen flux and then collected and stored under nitrogen. Selected IR data (ATR-IR, $\mathrm{cm}^{-1}$ ): 1435 (w), 1209 (w), 968 (m), 941 (m), 924 (s), $910(\mathrm{~m}), 836(\mathrm{~s}), 773$ (s), 698 (s), $585(\mathrm{w}), 548(\mathrm{w}), 483(\mathrm{w}), 415(\mathrm{~s})$. 
Elemental analysis for $\mathrm{Na}_{9}\left[\mathrm{Y}\left(\mathrm{W}_{5} \mathrm{O}_{18}\right)_{2}\right] \cdot 35 \mathrm{D}_{2} \mathrm{O}, \mathrm{D}_{70} \mathrm{YNa}_{9} \mathrm{O}_{71} \mathrm{~W}_{10}$, calcd (found): $\mathrm{D} 4.11$ (2.9), $\mathrm{Y}$ 2.7 (2.7), Na 6.1 (5.9), W 54 (54).

$\mathrm{Na} 9\left[\mathbf{L n}\left(\mathrm{W}_{5} \mathrm{O}_{18}\right)_{2}\right] \cdot 35 \mathrm{H}_{2} \mathrm{O}$ and $\mathrm{Na} 9\left[\mathrm{Y}_{(}\left(\mathrm{W}_{5} \mathrm{O}_{18}\right)_{2}\right] \cdot 35 \mathrm{D}_{2} \mathrm{O}, \quad \mathbf{L n}=\mathrm{Nd}, \mathrm{Dy}, \mathrm{Ho}, \mathrm{Er}$. Modification of the procedure used to synthesize $\mathbf{Y}$ and $\mathbf{Y}^{\mathbf{D}}$ to obtain the $\mathrm{Ln}=\mathrm{Nd}, \mathrm{Tb}, \mathrm{Dy}, \mathrm{Ho}$ and $\mathrm{Er}$ analogs involved the use of equivalent quantities of $\mathrm{Ln}\left(\mathrm{NO}_{3}\right)_{3}$ of the appropriate lanthanoid.

$\mathrm{Na}_{9}\left[\mathbf{N d}\left(\mathrm{W}_{5} \mathrm{O}_{18}\right)_{2}\right] \cdot 35 \mathrm{H}_{2} \mathrm{O}(\mathbf{N d})$. Yield after recrystallization $(2.2 \mathrm{~g}, 0.66 \mathrm{mmol}, 15 \%)$. Selected IR data $\left(\mathrm{KBr}, \mathrm{cm}^{-1}\right): 942$ (s), 891 (w), 845 (s), 784 (s), 704 (s), 578 (w), $542(\mathrm{~m}), 485$ (w), $416(\mathrm{~s})$.

$\mathbf{N a 9}\left[\mathbf{N d}\left(\mathbf{W}_{5} \mathbf{O}_{18}\right)_{2}\right] \cdot \mathbf{3 5 D}_{2} \mathbf{O}\left(\mathbf{N d}^{\mathrm{D}}\right)$. Selected IR data $\left(\right.$ ATR-IR, $\left.\mathrm{cm}^{-1}\right): 1441(\mathrm{w}), 1204(\mathrm{w})$, 965 (w), 921 (m), 828 (s), 774 (s), 691 (s), 586 (w), 539 (w), 476 (w), 408 (s). Elemental analysis for $\mathrm{Na}_{9}\left[\mathrm{Nd}\left(\mathrm{W}_{5} \mathrm{O}_{18}\right)_{2}\right] \cdot 35 \mathrm{D}_{2} \mathrm{O}, \mathrm{D}_{70} \mathrm{NdNa}_{9} \mathrm{O}_{71} \mathrm{~W}_{10}$, calcd (found): $\mathrm{D} 4.04$ (3.6), $\mathrm{Nd} 4.2$ (3.9), $\mathrm{Na}$ 6.0 (6.2), W 53 (55).

$\mathbf{N a 9}\left[\mathbf{T b}\left(\mathbf{W}_{5} \mathrm{O}_{18}\right)_{2}\right] \cdot 35 \mathrm{H}_{2} \mathrm{O}$ (Tb). Yield after recrystallization $(2.0 \mathrm{~g}, 0.59 \mathrm{mmol}, 13 \%)$. Selected IR data $\left(\mathrm{KBr}, \mathrm{cm}^{-1}\right)$ : $944(\mathrm{~m}), 844$ (s), 782 (m), 704 (s), 583 (w), 542 (m), 490 (w), 420 $(\mathrm{s})$.

$\mathbf{N a 9}\left[\mathbf{T b}\left(\mathbf{W}_{5} \mathrm{O}_{18}\right)_{2}\right] \cdot 35 D_{2} \mathbf{O}\left(\mathbf{T b}^{\mathbf{D}}\right)$. Selected IR data $\left(A T R-I R, \mathrm{~cm}^{-1}\right): 1649(\mathrm{w}), 1438(\mathrm{w})$, 1209 (w), 967 (m), 922 (s), 830 (s), 776 (s), 699 (s), 582 (w), 540 (w), 482 (m), 413 (s). Elemental analysis for $\mathrm{Na}_{9}\left[\mathrm{~Tb}\left(\mathrm{~W}_{5} \mathrm{O}_{18}\right)_{2}\right] \cdot 35 \mathrm{D}_{2} \mathrm{O}, \mathrm{D}_{70} \mathrm{TbNa}_{9} \mathrm{O}_{71} \mathrm{~W}_{10}$, calcd (found): $\mathrm{D} 4.0$ (3.4), Tb 4.6 (5.1), Na 6.0 (6.3), W 53 (55). 
$\mathbf{N a 9}\left[\operatorname{Dy}\left(\mathrm{W}_{5} \mathrm{O}_{18}\right)_{2}\right] \cdot 35 \mathrm{H}_{2} \mathrm{O}$ (Dy). Yield after recrystallization $(3.1 \mathrm{~g}, 0.91 \mathrm{mmol}, 20 \%)$. Selected IR data (KBr, cm-1): 934 (m), 848 (s), 790 (m), 710 (s), 584 (w), 544 (m), 492 (w), 423 (s). Elemental analysis for $\mathrm{Na}_{9}\left[\mathrm{Dy}\left(\mathrm{W}_{5} \mathrm{O}_{18}\right)_{2}\right] \cdot 35 \mathrm{H}_{2} \mathrm{O}, \mathrm{H}_{70} \mathrm{DyNa}_{9} \mathrm{O}_{71} \mathrm{~W}_{10}$, calcd (found): $\mathrm{H} 2.1$ (1.9), Dy 4.8 (4.4), Na 6.1 (6.2), W 54 (54).

$\mathrm{Na9}\left[\mathrm{Ho}\left(\mathrm{W}_{5} \mathrm{O}_{18}\right)_{2}\right] \cdot 35 \mathrm{H}_{2} \mathrm{O}(\mathrm{Ho})$. Yield after recrystallization $(3.4 \mathrm{~g}, 1.0 \mathrm{mmol}, 22 \%)$. Selected IR data $\left(\mathrm{KBr}, \mathrm{cm}^{-1}\right)$ : $936(\mathrm{~s}), 848(\mathrm{~s}), 781(\mathrm{~m}), 703(\mathrm{~s}), 582(\mathrm{w}), 542(\mathrm{~m}), 490(\mathrm{w}), 415$ (s).

$\mathrm{Na9}\left[\mathrm{Ho}_{\mathbf{2}}\left(\mathrm{W}_{5} \mathrm{O}_{18}\right)_{2}\right] \cdot \mathbf{3 5 D}_{2} \mathrm{O}\left(\mathbf{H o}^{\mathrm{D}}\right)$. Selected IR data $\left(\mathrm{ATR}-\mathrm{IR}, \mathrm{cm}^{-1}\right): 1435(\mathrm{w}), 1204(\mathrm{w})$, $966(\mathrm{~m}), 924$ (s), 830 (s), 768 (s), 683 (s), 583 (w), 545 (w), 487 (m), 413 (s). Elemental analysis for $\mathrm{Na}_{9}\left[\mathrm{Ho}\left(\mathrm{W}_{5} \mathrm{O}_{18}\right)_{2}\right] \cdot 35 \mathrm{D}_{2} \mathrm{O}, \mathrm{D}_{70} \mathrm{HoNa}_{9} \mathrm{O}_{71} \mathrm{~W}_{10}$, calcd (found): D 4.02 (3.3), Ho 4.7 (5.2), $\mathrm{Na}$ $5.9(6.2)$, W 53 (52).

$\mathrm{Na9}\left[\mathbf{E r}\left(\mathrm{W}_{5} \mathrm{O}_{18}\right)_{2}\right] \cdot 35 \mathrm{H}_{2} \mathrm{O}$ (Er) Yield after recrystallization (4.3 g, $\left.1.3 \mathrm{mmol}, 28 \%\right)$. Selected IR (KBr, cm $\left.{ }^{-1}\right) 945(\mathrm{~m}), 851$ (s), $779(\mathrm{~m}), 698$ (s), $583(\mathrm{w}), 545$ (m), 495 (w), $421(\mathrm{w})$.

$\mathbf{N a 9}\left[\mathbf{E r}\left(\mathrm{W}_{\mathbf{5}} \mathbf{O}_{18}\right)_{2}\right] \cdot \mathbf{3 5 D}_{\mathbf{2}} \mathbf{O}\left(\mathbf{E r}^{\mathbf{D}}\right)$. Selected IR data $\left(\mathrm{ATR}-\mathrm{IR}, \mathrm{cm}^{-1}\right): 1436(\mathrm{w}), 1208(\mathrm{w})$, 968 (m), 923 (s), 834 (s), 773 (s), 696 (s), 584 (w), 550 (w), 482 (m), 416 (s). Elemental analysis for $\mathrm{Na} 9\left[\operatorname{Er}\left(\mathrm{W}_{5} \mathrm{O}_{18}\right)_{2}\right] \cdot 35 \mathrm{D}_{2} \mathrm{O}, \mathrm{D}_{70} \mathrm{ErNa}_{9} \mathrm{O}_{71} \mathrm{~W}_{10}$, calcd (found): D 4.0 (3.6), Er 4.8 (4.8), Na 5.9 (6.2), W 53 (48).

Single Crystal X-ray Diffraction and Structure Solution. Diffraction measurements (Table S1) were performed on an Agilent Technologies SuperNova diffractometer using $\mathrm{CuK}_{\alpha}$ radiation $(\lambda=1.54184 \AA)$ at $90 \mathrm{~K}$. The collected data were processed with CrysAlisPro v171.37.35 software. ${ }^{60}$ Gaussian absorption corrections were applied for all compounds. Using 
the software Olex 2 v1.2, the structures were solved by direct methods using the SHELXT-2014 structure solution program and refined by full-matrix least-squares techniques on $F^{2}$ by using the SHELXL-2014 crystallographic software package. ${ }^{61-63}$ For each structure all atoms were refined with anisotropic displacement parameters, including the sodium cations and their coordinating water molecules; some of the oxygen atoms of $\left[\mathrm{Ln}\left(\mathrm{W}_{5} \mathrm{O}_{18}\right)_{2}\right]^{9-}$ moiety (Tb: O31; Dy: O29; Ho: O31; Er: O6; Nd: O17, O20', O35'), however, were restrained to near isotropic values during the refinement. None of the structures showed any significant disorder. The position of hydrogen atoms could not be determined in any of the structures, given the presence of tungsten atoms with very high atomic scattering factors.

Magnetic Measurements. Variable temperature magnetic susceptibility and magnetization measurements were performed with a Quantum Design MPMS-5 susceptometer, equipped with a $5 \mathrm{~T}$ magnet. Data were collected on powdered, dry crystals restrained in Vaseline in a gelatin capsule. Magnetic susceptibility data were collected with fields of $1 \mathrm{kOe}, 5$ $\mathrm{kOe}$, and $10 \mathrm{kOe}$. The diamagnetic susceptibility was measured for $\mathbf{Y}^{\mathbf{D}}$ and found to be very similar to the diamagnetic correction calculated from Pascal's constants, which was employed to correct the magnetic susceptibility, measured for $\mathbf{N d}^{\mathbf{D}}, \mathbf{T b}^{\mathbf{D}}, \mathbf{D y}, \mathbf{H o} \mathbf{D}^{\mathbf{D}}, \mathbf{E r}^{\mathbf{D}}$.

Inelastic Neutron Scattering. The inelastic neutron scattering measurements were carried out using the PELICAN time-of-flight spectrometer at the Australian Nuclear Science and Technology Organisation facilities in Lucas Heights. ${ }^{64}$ Each sample $\left(\mathbf{N d} \mathbf{d}^{\mathbf{D}}, \mathbf{T b}^{\mathbf{D}}, \mathbf{H o}^{\mathbf{D}}, \mathbf{E r}^{\mathbf{D}}\right.$, $\mathbf{Y}^{\mathbf{D}}$ ) was comprised of 2-4 g of deuterated coarsely ground, crystalline product sealed in a $1 \mathrm{~mm}$ double-wall aluminum can in order to give a $10 \%$ scatter and thus minimize multiple scattering. The background due to the empty sample can was subtracted and the data normalized to a vanadium standard. The INS spectrum of the diamagnetic $\mathbf{Y}^{\mathbf{D}}$ compound was collected at 10 and 
$50 \mathrm{~K}$ to estimate the phonon background. The sample was cooled using a displex type cryostat and data collected at multiple temperatures. PELICAN is primarily designed to work with cold neutrons; in the current experiment the primary configuration was for $4.74 \AA$ neutrons obtained via a PG002 monochromator with no beryllium filter and higher orders wavelengths removed by phasing the Fermi choppers, reaching a maximum energy transfer of $3.6 \mathrm{meV}(1.000 \mathrm{meV}=$ $\left.8.066 \mathrm{~cm}^{-1}\right)$. By an appropriate phasing of the Fermi choppers it was also possible to use the PG004 reflection $(\lambda / 2)$ at $2.37 \AA$ neutron wavelength without changing the physical configuration of the instrument, thus allowing us to reach up to $14.4 \mathrm{meV}$ in energy transfer. In both configurations the master and slave Fermi choppers were operated at $100 \mathrm{~Hz}$. This last feature is particularly important in order to observe CF excitations in molecules showing SMM behavior for which relatively large CF energy gaps are expected ${ }^{42,54}$ For each sample, data were collected both at $4.74 \AA$ and $2.37 \AA$ obtaining complementary information. Neutrons with a 4.74 $\AA$ wavelength, despite the narrower overall energy transfer range, offer better resolution (135 $\mu \mathrm{eV}$ at the elastic line) and better access to the neutron energy gain side of the spectrum, whereas neutrons with a $2.37 \AA$ wavelength gives access to a broader energy transfer range allowing the observation of otherwise inaccessible transitions on the energy loss side of the spectrum, but with lower resolution $(850 \mu \mathrm{eV}$ at the elastic line). The data were reduced using the following procedure: an empty can was subtracted and all data normalized to a vanadium standard. The data were then converted to $S(\boldsymbol{Q}, \omega)$. All manipulations and sample fitting were carried out using the LAMP software. ${ }^{65}$ Data were analyzed to determine whether any observed signals were spurious. Typical identification of a spurion is based on whether the signal is narrower than the instrumental resolution, does not obey detailed balance and displays no temperature dependence. Numerous effects can give rise to spurious scattering, such as scattering from sample 
environment and multiple scattering from components in the neutron beam. PELICAN is constructed and calibrated to minimize such effects, though it is impossible to remove them for all samples and sample environments. ${ }^{66}$

INS data analysis and fitting. The position and relative intensity of the magnetic peaks of $\mathbf{N d}^{\mathbf{D}}, \mathbf{T b}^{\mathbf{D}}, \mathbf{H o} \mathbf{D}^{\mathbf{D}}$, and $\mathbf{E r}^{\mathbf{D}}$ were determined by fitting the experimental INS spectra with Gaussian-shaped curves. The MCPHASE modelling suite was employed in fitting the experimental INS using a CF approach based on the Extended Stevens Operators formalism (Equation 1). ${ }^{67,68}$

$$
\widehat{H}_{C F}=\sum_{k=2,4,6} \sum_{q=-k}^{k} B_{k}^{q} \widehat{O}_{k}^{q}
$$

In first approximation the $D_{4 d}$ pseudo axial symmetry of $\left[\mathrm{Ln}\left(\mathrm{W}_{5} \mathrm{O}_{18}\right)_{2}\right]^{9-}$ suggests that the CF Hamiltonian can be simplified to the expression containing only the diagonal CF terms (Equation 2). ${ }^{69}$

$$
\widehat{H}_{C F}=B_{2}^{0} \hat{O}_{2}^{0}+B_{4}^{0} \hat{O}_{4}^{0}+B_{6}^{0} \hat{O}_{6}^{0}
$$

However, because of the actual $C_{1}$ point symmetry of the $\left[\mathrm{Ln}\left(\mathrm{W}_{5} \mathrm{O}_{18}\right)_{2}\right]^{9-}$ anion, the appropriate CF Hamiltonian that should be considered is that of Equation 1, containing 27 terms.

The relatively small number of magnetic excitations that we observe with INS (see below) necessarily prevents the fitting of all $27 \mathrm{CF}$ parameters of the full CF Hamiltonian (Equation 1). However, with the intent of reproducing only the effects of the low symmetry on the INS spectra, we employed a specific CF model Hamiltonian for each of $\mathbf{E r} \mathbf{r}^{\mathbf{D}}, \mathbf{H o}^{\mathbf{D}}$, and $\mathbf{T b}^{\mathbf{D}}$, with the minimum number of terms necessary to reproduce the experimentally observed low lying CF levels. ${ }^{32}$ These minimum sets of $\mathrm{CF}$ terms are comprised of the diagonal terms and, 
whenever necessary to interpret the INS data, the minimum number of appropriate off-diagonal terms.

The CF parameter sets of the truncated CF Hamiltonians for $\mathbf{E} \mathbf{r}^{\mathbf{D}}, \mathbf{H} \mathbf{o}^{\mathbf{D}}$ and $\mathbf{T b}^{\mathbf{D}}$ were optimized using the Simannfit fitting module of the MCPHASE modelling suite. ${ }^{67}$ The fitting strategy implemented in Simannfit uses a simulated annealing Monte Carlo method algorithm which varies the CF parameters to iteratively minimize the standard deviation between the simulated INS spectra and the Gaussian fitting of experimental INS spectra, taking into account both position and relative intensities of the peaks. ${ }^{70}$ During the iterative fitting procedure, the spectrum of the theoretical INS transition probabilities is convoluted with Gaussian functions of appropriate linewidth to generate the simulated INS spectrum which is in turn compared to the experimental one. The temperature dependence of magnetic susceptibility and the magnetic field dependence of the magnetization were then simulated from the optimized sets of CF parameters using the Easyspin software package. ${ }^{71}$

Other Measurements. Elemental analyses were performed by the Campbell Microanalytical Laboratory, University of Otago, New Zealand. Thermogravimetric analyses were performed on a Mettler Toledo thermal analyzer. Infrared spectra ( $\mathrm{KBr}$ disk or ATR) were recorded on a Bruker Tensor 27 FTIR spectrometer.

Theoretical Calculations The ab initio CASSCF/RASSI calculations were carried out using the Molcas 8.0 suite. $^{72-74}$ The structural input for the calculations for all compounds: Nd, Tb, Dy, Ho, and Er, were the Cartesian atomic coordinates obtained by the single crystal X-ray structural analyses carried out here, all at $90 \mathrm{~K}$. Two sets of calculations were carried out for each compound: (i) an initial treatment of an isolated $\left[\mathrm{Ln}\left(\mathrm{W}_{5} \mathrm{O}_{18}\right)_{2}\right]^{9-}$ polyanion was followed by (ii) more extensive calculations on a $\left[\mathrm{Ln}\left(\mathrm{W}_{5} \mathrm{O}_{18}\right)_{2}\right]^{9-}$ polyanion surrounded by point charges in 
the crystallographically determined positions, including the nine Na countercations per POM, in order to provide electroneutrality and to represent the electrostatic potential of the atoms in the crystal lattice. Mulliken charges obtained from (i) were employed in (ii) to assign fractional charges to $\mathrm{Ln}, \mathrm{O}$, and $\mathrm{W}$ atoms, while $\mathrm{Na}$ ions where assumed to be monopositive. For (ii) the point charges were placed at crystallographically-determined positions in a volume spanning $9 \times 9 \times 9$ unit cells $(81601$ point charges including 1457 Ln centers) for the isomorphous compounds Tb, Dy, Ho, and Er, and 7×7×7 unit cells (76785 point charges including $1371 \mathrm{Ln}$ centers) for $\mathbf{N d}$, with the ab initio treated $\mathrm{Ln}^{\mathrm{III}}$ ion kept at the center of the distribution. The size of the distribution was chosen so that the ab initio energies had minimal variation $(<0.05 \mathrm{meV})$ upon further increase in the number of layers of unit cells considered. The point symmetry for the isolated $\left[\mathrm{Ln}\left(\mathrm{W}_{5} \mathrm{O}_{18}\right)_{2}\right]^{9-}$ anion used in all the ab initio calculations was $C_{1}$, as crystallographically determined. In terms of computational demand, any attempt to use full atomic orbital basis sets to describe the $\mathrm{W}$ atoms in the $\left\{\mathrm{W}_{5} \mathrm{O}_{18}\right\}$ POM ligand is extremely challenging due to the large number of electrons these atoms possess. To reduce the computational load, all $\mathrm{W}$ atoms were represented with an Ab Initio Model Potential (AIMP) basis set, using the contraction suggested by the Molcas developers (W.ECP.Casarrubios.13s10p9d5f.3s3p4d2f.12e-CG-AIMP). ${ }^{75,76}$ In this way only the valence electrons of W are treated quantum mechanically, whereas the core electrons are "frozen" and represented by an effective potential. For other elements $(\mathrm{Ln}, \mathrm{O})$ the standard Molcas relativistic ANO-RCC libraries were employed, with TZP and DZP quality for Ln and O, respectively.

Different modules of Molcas (SEWARD, RASSCF, RASSI) were sequentially employed to determine the full ab initio electrostatic and strongly spin-orbit coupled eigenvalues and eigenvectors together with treatment of static electronic correlation via complete active space 
methods. The computation of the mono and bi-electronic integrals was performed with SEWARD and a Cholesky decomposition of bielectronic integrals was employed with a $10^{-8}$ threshold to save disk space. The spin-only wavefunctions and corresponding energies were optimized using the Complete Active Space Self-Consistent Field (CASSCF) method supplied by the module RASSCF. ${ }^{39}$ The active space for the CASSCF method corresponded to the $4 f$-type orbitals of the $\mathrm{Ln}^{\mathrm{III}}$ ion in $\mathbf{N d}, \mathbf{T b}, \mathbf{D y}, \mathbf{H o}$, and $\mathbf{E r}$ with 3, 8, 9, 10, and 11 electrons respectively. The optimization of the spin-only wavefunctions was carried out on the manifolds corresponding to spin multiplicity 4 and 2 (Nd), 7, 5, 3, and 1 (Tb), 6, 4, and 2 (Dy), 5, 3, and 1 (Ho), 4 and 2 (Er). ${ }^{39}$ The module RASSI (Restricted Active Space State Interaction) was employed to introduce the strong spin-orbit interaction that characterizes lanthanoid atoms via the DouglasKroll-Hess Hamiltonian in the mean-field approximation. ${ }^{40}$ Due to calculation power limitations only a number of selected states were allowed to interact in the RASSI step, namely for Nd 35 quartets (out of 35) and 89 doublets (out of 112), for Tb 7 septets (out of 7), 84 quintets (out of 140) and 58 triplets (out of 588) (none of the 490 singlets were included in the calculations because of their high energy), for Ho 30 quintets (out of 35), 60 triplets (out of 210), and 17 singlets (out of 196), for Dy 21 sextets (out of 21), 108 quartets (out of 224), 17 doublets (out of 490), and finally 35 quartets (out of 35) and 89 doublet (out of 112) for Er.

Ab initio calculated magnetic properties and INS spectral simulations. The SINGLE_ANISO module of Molcas $8.0^{41,77}$ was used to calculate the magnetic susceptibility and the magnetization from CASSCF/RASSI results. The components of the RASSI wavefunctions corresponding to the ground state multiplet projected onto a $(2 J+1)$-dimensional pseudo-spin basis set, together with the associated ab initio CF parameters (Table S2), were calculated employing a projection technique implemented in SINGLE_ANISO. The matrix 
elements of the all-electron spin and angular momentum operators between low-lying CASSCF/RASSI wavefunctions corresponding to the CF levels were calculated to obtain the INS transition probabilities. These transition probabilities were then convoluted with Gaussian curves of appropriate linewidth to calculate the INS peak intensities in the theoretical INS spectra reported herein.

\section{Results and Discussion}

Syntheses. The synthesis of sodium salts of the lanthanoid decatungstate was based on the procedure reported by Peacock and Weakley. ${ }^{50}$ The crude microcrystalline product obtained in ca. 50\% yield (based on W) by keeping the reaction solution at $5{ }^{\circ} \mathrm{C}$ is recrystallized from warm water, yielding the target product in yields ranging from $c a$. $10 \%$ to $30 \%$, depending on the lanthanoid ion. Slow evaporation at room temperature affords crystals of $\mathbf{T b}, \mathbf{D y}, \mathbf{H o}, \mathbf{E r}$, and Nd. Rapid crystallization at $5{ }^{\circ} \mathrm{C}$ gives rise to another phase of the same compound for $\mathrm{Tb}$, Dy, Ho, and $\mathrm{Er}^{78}$ which can be avoided by slow evaporation of dilute solutions at room temperature. ${ }^{32}$ A common byproduct of this synthetic procedure is sodium paratungstate, which is much less soluble than the sodium salts of the target compound and is removed in the recrystallization step.

The deuterated compounds $\mathbf{T} \mathbf{b}^{\mathbf{D}}, \mathbf{H} \mathbf{o}^{\mathbf{D}}, \mathbf{E} \mathbf{r}^{\mathbf{D}}$ and $\mathbf{N d}^{\mathbf{D}}$ were obtained by recrystallizing the corresponding hydrated product from $\mathrm{D}_{2} \mathrm{O}$. Prior to crystallization from $\mathrm{D}_{2} \mathrm{O}$, the hydrous samples were heated under reduced pressure at $150{ }^{\circ} \mathrm{C}$ to dehydrate them as much as possible. After recrystallization from $\mathrm{D}_{2} \mathrm{O}$, the samples were sealed and kept under $\mathrm{N}_{2}$ to avoid rapid 
$\mathrm{D}_{2} \mathrm{O} / \mathrm{H}_{2} \mathrm{O}$ exchange with atmospheric moisture and crystals of the deuterated products were obtained within $24 \mathrm{~h}$.

Structures. Single crystal X-ray structural analyses (Table S1) indicate that compounds Tb, Dy, Ho, Er, and their corresponding deuterated versions, are isomorphous. Among these compounds, only the structure of $\mathbf{H o}$ is previously unreported; the structures of the other compounds are already known..$^{32,54,79,80}$ The structure of Nd and its isomorphous deuterated version, these are different from those of the other analogs; neither has been reported previously. All compounds are comprised of isostructural $\left[\mathrm{Ln}\left(\mathrm{W}_{5} \mathrm{O}_{18}\right)\right]^{9-}$ polyanions surrounded by a

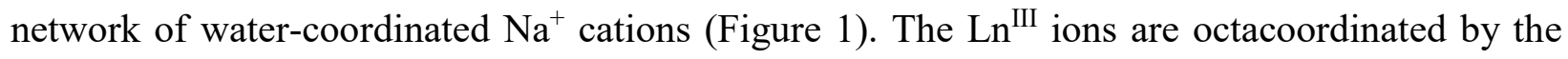
four oxygen atoms provided by each of the monolacunary $\left\{\mathrm{W}_{5} \mathrm{O}_{18}\right\}$ Lindqvist moieties in a slightly distorted square antiprismatic geometry providing a $D_{4 d}$ pseudo-symmetry of the Ln center. The angle $(\alpha)$ formed by the Ln-O bonds and the axis passing through the centroids of the square faces of the antiprism is larger than the magic angle $\left(54.74^{\circ}\right)$, affording a compressed square antiprismatic geometry and the O-donor atoms are therefore considered equatorial (Table S3). ${ }^{2}$ While $\mathbf{T b}$, Dy, $\mathbf{H o}$, and $\mathbf{E r}$ contain a single $\left[\mathrm{Ln}\left(\mathrm{W}_{5} \mathrm{O}_{18}\right)\right]^{9-}$ anion in the asymmetric unit, two crystallographically independent $\left[\mathrm{Nd}\left(\mathrm{W}_{5} \mathrm{O}_{18}\right)\right]^{9-}$ anions are present in the asymmetric unit of Nd. 

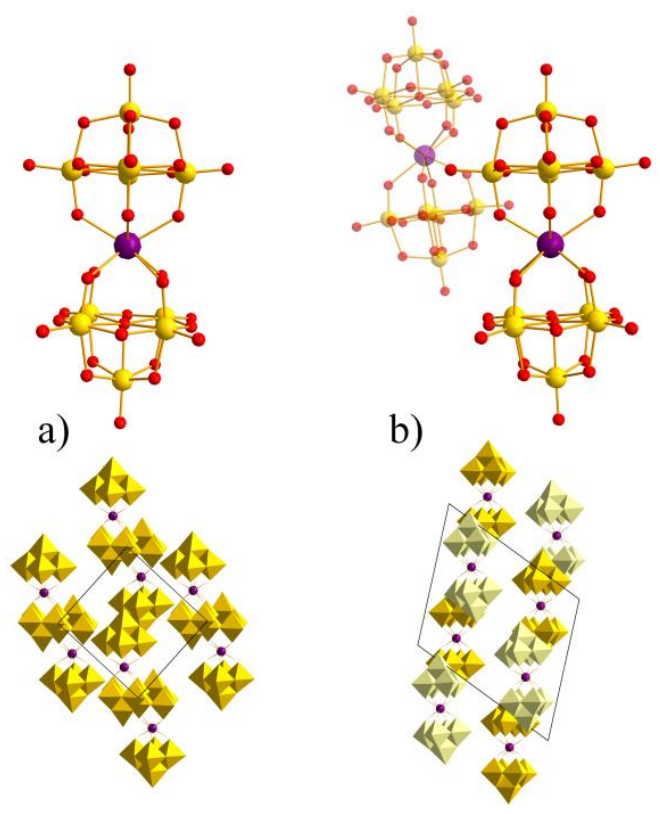

Figure 1. Ball and stick representations (top) and crystal packing diagram using polyhedral representations (bottom) of a) the $\left[\mathrm{Tb}\left(\mathrm{W}_{5} \mathrm{O}_{18}\right)_{2}\right]^{9-}$ polyanion in $\mathbf{T b}$ (packing viewed along the $c$ axis) and b) the crystallographically distinct $\left[\mathrm{Nd}\left(\mathrm{W}_{5} \mathrm{O}_{18}\right)_{2}\right]^{9-}$ polyanions in $\mathbf{N d}$ (packing viewed along the $a$ axis). Color code: violet, Ln; yellow: W, red: O; yellow octahedra: $\mathrm{WO}_{6}$ units. Sodium cations and coordinating water molecules are omitted for clarity.

The deviations from $D_{4 d}$ symmetry of the geometry of the first coordination sphere of the central $\mathrm{Ln}^{\mathrm{III}}$ ion can be described using the absolute average deviation from $45^{\circ}\left(\mathrm{AD}(\phi)_{45}=\right.$ $\left.\frac{\sum_{i=1}^{4}\left|\phi_{i}-45\right|}{4}\right)$ of the dihedral angles $\phi_{i}$ generated by the four independent pairs of O-Ln-O planes and in terms of the angle $\varphi$ between the normal vectors to the square faces of the antiprism, which in the ideal geometry is $0^{\circ}$ (Figure S1, Figure S2 and Table S3). The essentially constant value of $\operatorname{AD}(\phi)_{45}$ across the isomorphous structures $\mathbf{T b}, \mathbf{D y}, \mathbf{H o}$ and $\mathbf{E r}$ suggests that the ionic radius of the $\mathrm{Ln}^{\mathrm{III}}$ ion has little effect and that it is the crystal packing that dictates the magnitude 
of $\operatorname{AD}(\phi)_{45}$. The value of angle $\varphi$ for $\mathbf{T b}, \mathbf{D y}, \mathbf{H o}$ and $\mathbf{E r}$ is more dependent on the $\mathrm{Ln}^{\mathrm{III}}$ ion, with a tendency to decrease with ionic radii in going from $\mathrm{Tb}$ to Er. Continuous shape measurements carried out with the software SHAPE 2.1 confirm that in all structures a square antiprism is the closest ideal coordination geometry to that observed for the $\mathrm{Ln}^{\mathrm{III}}$ ions. ${ }^{81,82}$ The two crystallographically distinct $\left[\mathrm{Nd}\left(\mathrm{W}_{5} \mathrm{O}_{18}\right)_{2}\right]^{9-}$ polyanions in $\mathbf{N d}$ are isostructural to each other and to those in $\mathbf{T b}, \mathbf{D y}, \mathbf{H o}$, and $\mathbf{E r}$. The two local coordination environments of the $\mathrm{Nd}^{\mathrm{III}}$ differ mainly in the angle $\varphi$ between the normal vectors of the square faces of the square antiprism, whereas the $\mathrm{AD}(\phi)_{45}$ value is the same (Table S3). The average Ln-O distance across all the reported compounds (Table S3) decreases in going from Nd to $\mathbf{E r}$, following the lanthanoid contraction. The shortest intermolecular $\operatorname{Ln} \cdots \operatorname{Ln}$ distance is $9.9 \AA$ for $\mathbf{N d}$ and $11.2 \AA$ for $\mathbf{T b}, \mathbf{D y}$, Ho and Er.

Characterization of bulk samples. A suite of techniques was employed to check the purity of bulk samples of $\mathbf{N d}^{\mathbf{D}}, \mathbf{T} \mathbf{T}^{\mathbf{D}}, \mathbf{D y}, \mathbf{H o} \mathbf{D}^{\mathbf{D}}$ and $\mathbf{E} \mathbf{r}^{\mathbf{D}}$. Infrared spectra in the $1000-400 \mathrm{~cm}^{-1}$ region matched the literature data reported for these compounds (Figure S3). ${ }^{42,54}$ Thermogravimetric analysis (TGA) data show a decrease in mass due to the loss of the hydration water molecules ranging from 8 to 17 per cent, which although lower than the theoretical value corresponding to 35 water molecules ( $c a .20 \%$ ), is still consistent with the water molecules observed crystallographically (Figure S4). The lower value recorded with TGA is most likely due to the fact that the samples are finely ground and exposed to air before being analyzed, causing the partial loss of crystallization water molecules. The effectiveness of the deuteration of INS samples was verified by the presence of the diagnostic infrared peaks of $\mathrm{DHO}$ and $\mathrm{D}_{2} \mathrm{O}$ bending at $c a .1440$ and $1200 \mathrm{~cm}^{-1}$ respectively in the infrared spectra, compared to the $\mathrm{H}_{2} \mathrm{O}$ bending peak at $c a$. $1650 \mathrm{~cm}^{-1}$ (Figure S5). In order to avoid the $\mathrm{H}_{2} \mathrm{O}$ contamination that comes 
with $\mathrm{KBr}$ pellets, the IR spectra were collected on bulk powders using an attenuated total reflectance (ATR) adapter. The static and dynamic magnetic properties of the samples were measured both to confirm the purity of the samples and to confirm that the SMM properties are retained in the deuterated compounds (Figures S6-S10). The values of $\chi_{M} T$ at $300 \mathrm{~K}$ for $\mathbf{N d}^{\mathbf{D}}$ (1.64 emu mol $\left.{ }^{-1} \mathrm{~K}\right), \mathbf{T b}^{\mathbf{D}}\left(11.90 \mathrm{emu} \mathrm{mol}{ }^{-1} \mathrm{~K}\right), \mathbf{D y}\left(14.08 \mathrm{emu} \mathrm{mol}{ }^{-1} \mathrm{~K}\right), \mathbf{H o}^{\mathbf{D}}\left(13.87 \mathrm{emu} \mathrm{mol}{ }^{-1}\right.$ $\mathrm{K})$, and $\mathbf{E r}^{\mathbf{D}}\left(11.24 \mathrm{emu} \mathrm{mol}^{-1} \mathrm{~K}\right)$ are in good agreement with the free ion values $\left(\mathrm{Nd}^{\mathrm{III}}\right.$ : [Xe] $4 f^{3}$, ${ }^{4} \mathrm{I}_{9 / 2}, \mathrm{~g}_{\mathrm{J}}=8 / 11,1.64 \mathrm{emu} \mathrm{mol}^{-1} \mathrm{~K} ; \mathrm{Tb}^{\mathrm{III}}:[\mathrm{Xe}] 4 f^{8},{ }^{7} \mathrm{~F}_{6}, \mathrm{~g}_{\mathrm{J}}=3 / 2,11.81 \mathrm{emu} \mathrm{mol}{ }^{-1} \mathrm{~K} ; \mathrm{Dy}{ }^{\mathrm{IIII}}$ : [Xe $4 f^{9}$, ${ }^{6} \mathrm{H}_{15 / 2}, \mathrm{~g}_{\mathrm{J}}=4 / 3,14.17 \mathrm{emu} \mathrm{mol}^{-1} \mathrm{~K} ; \mathrm{Ho}^{\mathrm{IIII}}:[\mathrm{Xe}] 4 f^{f 0},{ }^{5} \mathrm{I}_{8}, \mathrm{~g}_{\mathrm{J}}=5 / 4,14.06 \mathrm{emu} \mathrm{mol}{ }^{-1} \mathrm{~K} ; \mathrm{Er}^{\mathrm{IIII}}$ :

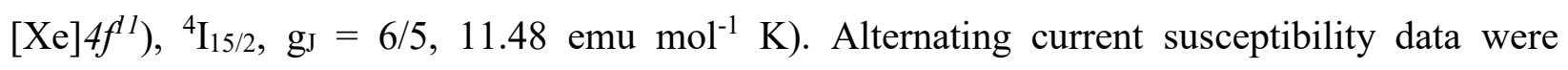
collected for the members of the series reported as SMMs by Coronado et al. $\left(\mathbf{E r}^{\mathbf{D}}, \mathbf{H o}^{\mathbf{D}}, \mathbf{N d}^{\mathbf{D}}\right)$ showing that slow relaxation of the magnetization is retained in the deuterated samples (Figures S10-S10).

\section{Inelastic neutron scattering, ab initio calculations, and magnetic properties.}

$\mathrm{Na9}\left[\mathbf{E r}\left(\mathbf{W}_{5} \mathbf{O}_{18}\right)_{2}\right]$ (Er). Compound $\mathbf{E r}$ exhibits SMM behavior in zero applied external magnetic field with an experimental effective energy barrier to magnetization reversal of 4.8 $\left.\mathrm{meV}\left(39 \mathrm{~cm}^{-1}\right)\right)^{54}$ The INS spectra of $\mathbf{E r}^{\mathbf{D}}$ measured with both neutron wavelengths (Figure 2, Figures S11-S14) exhibit two sharp peaks at energies of 5.79(3) and 7.26(3) meV (labeled peaks I and II) at the base temperature (5 K). The temperature and the wave vector $(\boldsymbol{Q})$ dependencies (Figure S15), as well as comparison with the INS spectra of the diamagnetic analog $\mathbf{Y}^{\mathbf{D}}$ (Figure S16), indicate that peaks I and II are CF magnetic excitations from the ground state ("cold" transitions). At $50 \mathrm{~K}$ a third feature (peak III) becomes evident at 9.22(4) meV. In fulfillment of the detailed balance principle $\left(\mathrm{S}(-\boldsymbol{Q},-\omega)=\mathrm{S}(\boldsymbol{Q}, \omega) e^{-\frac{\hbar \omega}{k T}}\right)$, the three peaks are all mirrored on the 
energy gain side (negative energies) of the spectra with better resolution achieved with a neutron wavelength of $4.74 \AA$. The possibility that peak III is a phonon signal is ruled out by comparison with the spectra of $\mathbf{Y}^{\mathbf{D}}$ and peak III is assigned as a CF transition from an excited state ("hot" transition). The spectrum of the diamagnetic $\mathbf{Y}^{\mathbf{D}}$ analog, together with the temperature dependence, are also useful in assigning the features in the $1.5-4 \mathrm{meV}$ region as phonon signals.

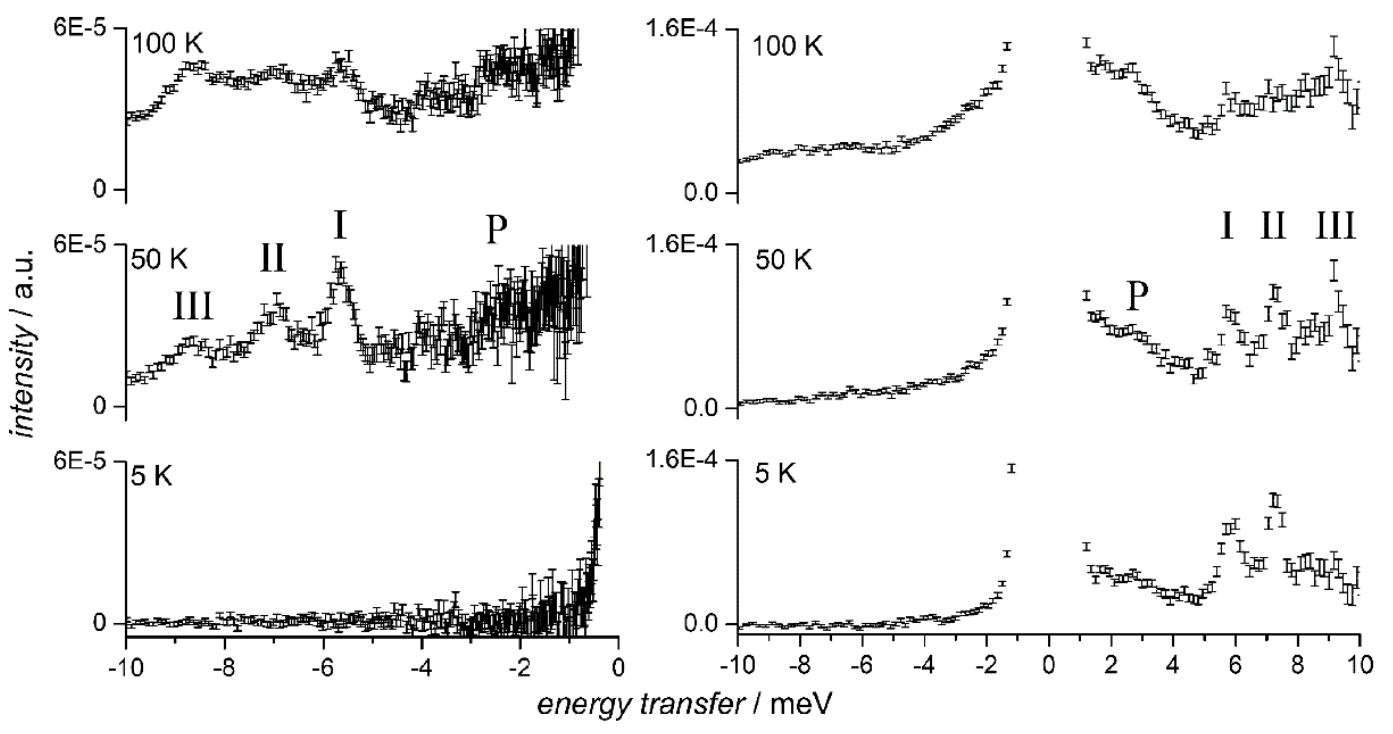

Figure 2. Variable temperature (5, 50 and $100 \mathrm{~K}$ ) INS spectra of $\mathbf{E r}^{\mathbf{D}}$. Left: $4.74 \AA$ A neutron wavelength spectra with integration over the whole $Q$ range. Right: $2.37 \AA$ neutron wavelength spectra with integration over the whole $Q$ range and $0.15 \mathrm{meV}$ binning interval. Positive numbers correspond to neutron energy loss. Magnetic transitions are labelled with Roman numerals and phonon signals designated as "P". 
The low-lying CF split states of the $J=15 / 2$ ground state of $\mathbf{E r}$ have been reported by Coronado et al. ${ }^{53-55}$ (Figure 3, right). The degree of admixture of the eigenfunctions of the Kramers doublets (KDs) depends on the form of the CF Hamiltonian employed by the various models, however Coronado and coworkers describe the ground state as an essentially $M_{J}= \pm 13 / 2$ and the first, second and third excited states as $M_{J}= \pm 1 / 2, M_{J}= \pm 15 / 2$, and $M_{J}= \pm 3 / 2$, respectively. The energy barrier to magnetization reversal determined by an Arrhenius plot is reported to be $4.8 \mathrm{meV} .{ }^{36,42,54,55}$

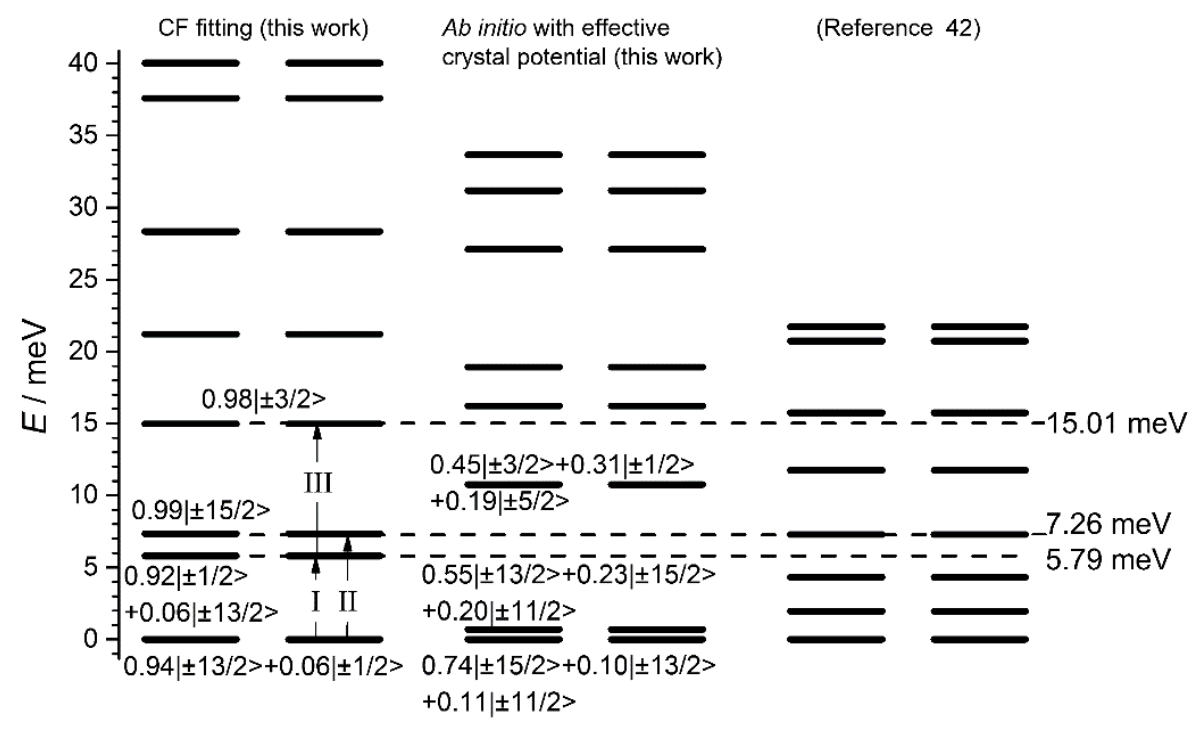

Figure 3. Energy level diagram for $\mathbf{E r}^{\mathbf{D}}$. Dashed lines indicate the energy levels experimentally observed with INS. Arrows correspond to the assignment of the CF transitions to the observed INS peaks. Comparison with energies reported by Coronado et l. $^{42}$ is provided.

In an initial effort to elucidate the electronic structure of $\mathbf{E r}$ and assign the INS transitions, we performed ab initio calculations on first the isolated $\left[\mathrm{Ln}\left(\mathrm{W}_{5} \mathrm{O}_{18}\right)_{2}\right]^{9-}$ polyanion 
and then including surrounding Mulliken partial point charges based on the crystallographic positions of the atoms in the lattice (see experimental ab initio section). Incorporating the electrostatic effects of the crystal lattice into the calculations in this way significantly affects the resulting energies and composition of wavefunctions corresponding to the CF split ground state $J$ $=15 / 2$ of Er ${ }^{\mathrm{III}}$. The energy levels are stabilized with respect to the ground state (Table S4-S5) and the ground state wavefunction, as determined by decomposition of the ab initio wavefunction in the reference system of the ground state $g$-tensor (Figure S17-S18), changes from an almost pure $M_{J}= \pm 15 / 2$ projection of the total angular momentum $(99 \%| \pm 15 / 2\rangle)$ obtained from calculations on the isolated polyanion, to an increased degree of admixture of $M_{J}$ values (Table S6-S7). The calculations incorporating electrostatic effects of the crystal lattice predict (Figure 3) a ground state $\mathrm{KD}$ and a first excited $\mathrm{KD}$ energetically close (ca. $0.7 \mathrm{meV}$ ), both characterized by a strong admixture of high $M_{J}$ states (ground state: $\left.74 \% \pm 15 / 2\right\rangle+10 \%$ $| \pm 13 / 2\rangle+11 \%| \pm 11 / 2\rangle ; 1$ st excited state: $23 \%| \pm 15 / 2\rangle+54 \%| \pm 13 / 2\rangle+20 \%| \pm 11 / 2\rangle)$ and separated by a large gap (ca. $10 \mathrm{meV})$ from the second excited $\mathrm{KD}(19 \%| \pm 5 / 2\rangle+45 \%| \pm 3 / 2\rangle+31 \%$ $| \pm 1 / 2\rangle)$. These calculations also improve the simulated $2 \mathrm{~K}$ limit of $\chi_{M} T$, which better approaches the experimental value of $c a .7 \mathrm{emu} \mathrm{mol}^{-1} \mathrm{~K}$, and slightly improve the low magnetic field behavior of the magnetization curve, indicating that the ground state composition is better reproduced when the electrostatic effects of the crystal lattice are included (Figure S19-S20). However the overall temperature dependence of $\chi_{M} T$ in the $2-150 \mathrm{~K}$ range, as well as the magnetization curve at $2 \mathrm{~K}$ for magnetic fields stronger than $5 \mathrm{kOe}$, are not well reproduced (Figure S19-S20), suggesting that the eigenvectors and eigenvalues calculated ab initio are not in good agreement with the microstates of the $J=15 / 2$ ground state manifold of Er. It is noteworthy that similar discrepancies between experimental and ab initio CASSCF calculated 
low temperature $\chi_{M} T$ values and magnetization isotherms have been reported for other $\mathrm{Er}^{\mathrm{III}}$ compounds. ${ }^{34,83}$ These discrepancies appear to be particular for $\mathrm{Er}^{\mathrm{III}}$ among the trivalent lanthanoid ions, and could be due to the limited size of the atomic basis set for $\mathrm{Er}^{\mathrm{III}}$ or to a contribution of dynamical electron correlation that is not accounted for in CASSCF/RASSI

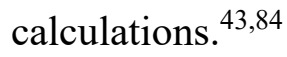

Utilizing an alternative CF approach, a satisfactory rationalization of the INS transitions observed for $\mathbf{E r}^{\mathbf{D}}$ can be achieved by fitting the experimental INS data to the parameters of a simplified CF Hamiltonian based on Extended Stevens Operators. ${ }^{68}$ The fitting of the experimental INS data was carried out using as a background the phonon spectra of $\mathbf{Y}^{\mathbf{D}}$ collected at $10 \mathrm{~K}$ and $50 \mathrm{~K}$ (Figure S16) with the $\mathbf{Y}^{\mathbf{D}}$ data at $10 \mathrm{~K}$ data scaled to match the intensity of the phonon region in $\mathbf{E r}^{\mathbf{D}}$ at $5 \mathrm{~K}$. On the basis of a purely axial CF Hamiltonian (Equation 2) it is not possible to rationalize the observed INS peaks. Assuming the electronic structure proposed for $\mathbf{E r}^{\mathbf{D}}$ by Coronado and coworkers, ${ }^{42,54}$ the cold transitions allowed by the $\Delta M_{J}= \pm 1$ INS selection rule $^{85}$ would be $M_{J}= \pm 13 / 2 \rightarrow M_{J}= \pm 15 / 2$ and $M_{J}= \pm 13 / 2 \rightarrow M_{J}= \pm 11 / 2$. It was however impossible to fit a set of CF parameters to Equation 2 so that the theoretical energies of the $M_{J}=$ $\pm 13 / 2 \rightarrow M_{J}= \pm 15 / 2$ and $M_{J}= \pm 13 / 2 \rightarrow M_{J}= \pm 11 / 2$ transitions, as well as the relative intensities, are the same as those of the two experimentally observed cold INS transitions (peaks I and II). As the ab initio calculations qualitatively indicate that the low lying CF levels are strong admixtures of different $M_{J}$ states, and considering that low symmetry effects have been already proven to play a key role in the physics of the members of this family of Ln-POMs, ${ }^{25,32,55}$ we pursued the hypothesis that the INS spectrum of $\mathbf{E r}^{\mathbf{D}}$ could be explained only by considering a certain degree of admixture between the ground state and the first excited states. Still referring to the electronic structure of $\mathbf{E r}^{\mathbf{D}}$ proposed by Coronado and coworkers, ${ }^{42,54}$ a good candidate as 
the energy level responsible for one of the two cold INS transitions is the first excited state, nominally $M_{J}= \pm 1 / 2$. The cold INS transition $M_{J}= \pm 13 / 2 \rightarrow M_{J}= \pm 1 / 2$ is forbidden in a strict $D_{4 d}$ axial symmetry, but it becomes allowed after introducing the extra diagonal $B_{6}^{6} \hat{O}_{6}^{6}$ term into the simplified CF Hamiltonian (Equation 3).

$$
\widehat{H}_{C F}=B_{2}^{0} \widehat{O}_{2}^{0}+B_{4}^{0} \widehat{O}_{4}^{0}+B_{6}^{0} \hat{O}_{6}^{0}+B_{6}^{6} \widehat{O}_{6}^{6}
$$

It must be noted that the $B_{6}^{6} \hat{O}_{6}^{6}$ term is not the only one of the 24 non-axial harmonics in the potential of a low-symmetry complex that can connect $M_{J}= \pm 13 / 2$ and $M_{J}= \pm 1 / 2$. This offdiagonal CF parameter has thus to be regarded as an effective parameter that incorporates all of the non-diagonal contributions that render allowed the transition between the ground state and the first excited Kramers doublet by strongly connecting the $M_{J}= \pm 13 / 2$ and $M_{J}= \pm 1 / 2$ states. The fitting of the experimental INS data to Equation 3 affords a set of 4 CF parameters (Table 1) that correctly reproduces the energies of the INS peaks and their relative intensities at 5 and $50 \mathrm{~K}$ (Figure 4). This same set of CF parameters reproduces very well the experimental temperature dependence of $\chi_{M} T$ and the magnetization as function of the magnetic field at $2 \mathrm{~K}$ (Figure 4) for Er $\mathbf{r}^{\mathbf{D}}$. The overall splitting of the of the $J=15 / 2$ ground state manifold obtained from our simplified model is ca. $40 \mathrm{meV}$ (Figure 3), which is larger than the ca. $20 \mathrm{meV}$ range obtained by Coronado et al. using an effective point charge model. ${ }^{42}$

The low lying CF levels obtained from our very simple model CF Hamiltonian are a mixed $M_{J}= \pm 13 / 2(94 \%| \pm 13 / 2\rangle+6 \%| \pm 1 / 2\rangle)$ ground state separated by $5.8 \mathrm{meV}$ from a mixed $M_{J}= \pm 1 / 2$ state $(94 \%| \pm 1 / 2\rangle+6 \%| \pm 13 / 2\rangle)$. The second and the third excited levels are a $99 \%$ $| \pm 15 / 2\rangle$ and a $98 \%| \pm 3 / 2\rangle$ state separated from the ground state by 7.26 and $15.01 \mathrm{meV}$, respectively. Within our simplified CF model (Figure 3), INS peaks I and II correspond to the 
transitions from the ground state to the first and second excited states, respectively, and the energy of these CF levels is obtained from the position of the INS peaks (5.79 and 7.26 meV). Peak III corresponds instead to a hot transition from the first to the third excited KD and the experimental energy of the third excited CF level $(15.01 \mathrm{meV})$ is obtained as the sum of the energy of the emitting level $(5.79 \mathrm{meV})$ with the energy of the transition corresponding to peak III (9.22 meV).

Table 1. CF parameters (meV) obtained from fitting INS data for $\mathbf{H o} \mathbf{o}^{\mathbf{D}}, \mathbf{T b}^{\mathbf{D}}$, and $\mathbf{E r}^{\mathbf{D}}$ to the eappropriate simplified CF Hamiltonians.

\begin{tabular}{|c|c|c|c|c|c|c|c|}
\hline & & $B_{2}^{0}$ & $B_{2}^{2}$ & $B_{4}^{0}$ & $B_{4}^{4}$ & $B_{6}^{0}$ & $B_{6}^{6}$ \\
\hline Er & & $-0.09131(1)$ & $a$ & $-7.344(4) \times 10^{-4}$ & $a$ & $1.067(4) \times 10^{-5}$ & $2.70(5) \times 10^{-5}$ \\
\hline \multirow[t]{2}{*}{ Ho } & Set 1 & $0.102(3)$ & $a$ & $5.62(9) \times 10^{-4}$ & $a$ & $-6.68(3) \times 10^{-6}$ & $a$ \\
\hline & Set 2 & $0.133(1)$ & $a$ & $5.25(4) \times 10^{-4}$ & $a$ & $-6.61(2) \times 10^{-6}$ & $a$ \\
\hline $\mathbf{T b}$ & & $0.705(1)$ & $0.0250(3)$ & $a$ & $-8.5(2) \times 10^{-4}$ & $a$ & $a$ \\
\hline
\end{tabular}

${ }^{a}$ parameter not required to fit low-lying energy levels involved in observed INS transitions 

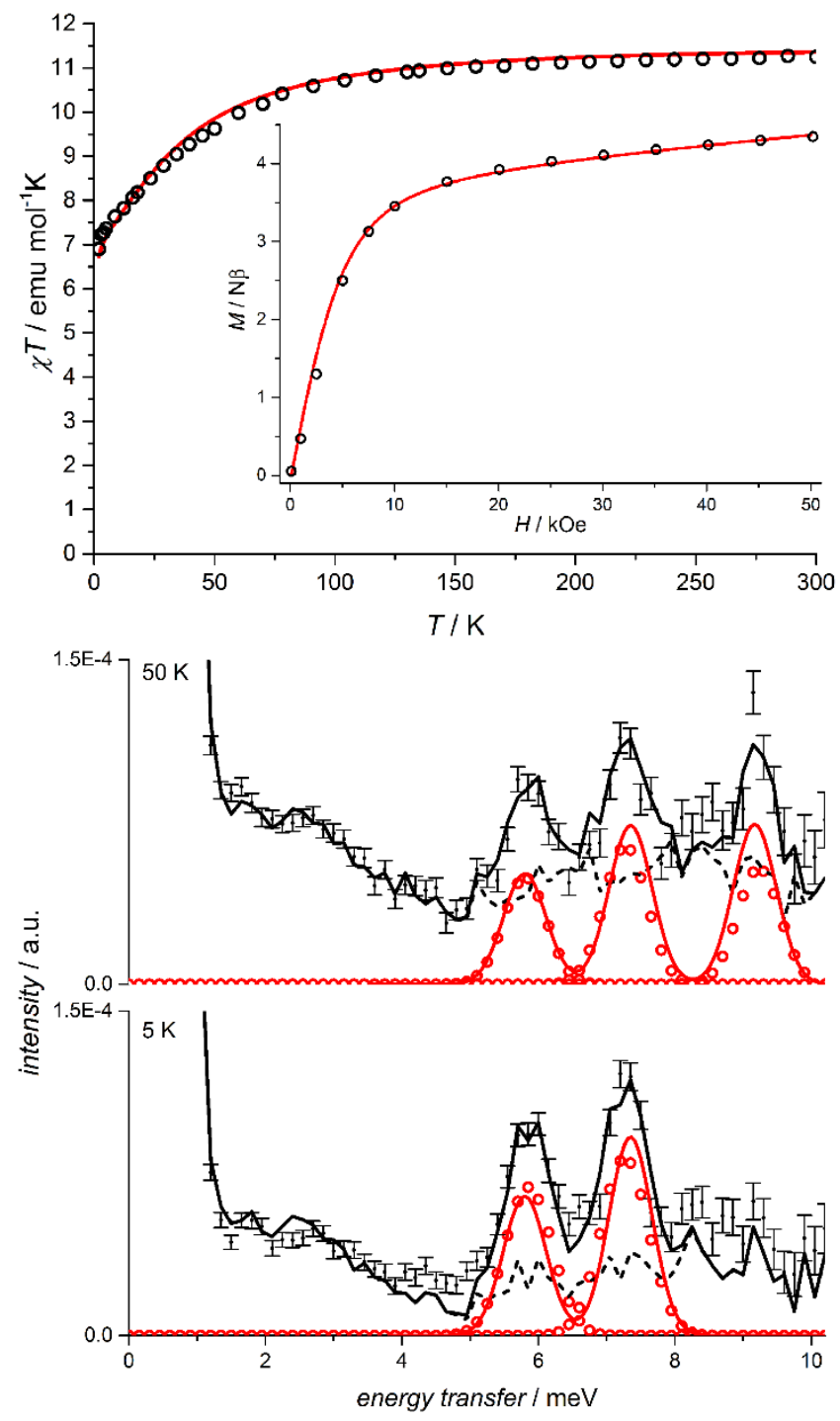

Figure 4. Top: Experimental (black circles) and calculated using the CF parameters from Table 1 (red line) magnetic properties for $\mathbf{E r}^{\mathbf{D}}$. Main: $T$ dependence of $\chi_{M} T$ product in the 2-300 $\mathrm{K}$ range. Inset: magnetization as function of magnetic field at $2 \mathrm{~K}$. Bottom: experimental (black) and simulated (red) ErD INS spectra. Experimental INS spectra (points with error bars); experimental scaled phonon background of $\mathbf{Y}^{\mathbf{D}}$ (dashed black line); sum of experimental scaled phonon background of $\mathbf{Y}^{\mathbf{D}}$ and individual magnetic peak contributions as Gaussian curves (solid black line); individual magnetic contributions as Gaussian curves (red open circles); simulation of the INS spectra using the optimized set of CF parameters from Table 1 (red line). 
The effective energy barrier to magnetization reversal for $\mathbf{E r}$ determined by ac susceptibility measurements is $c a .4 .8 \mathrm{meV},{ }^{54}$ which is lower than the first excited $\mathrm{KD}$ energy (ca. $5.8 \mathrm{meV})$ as determined by INS. This indicates that in addition to the Orbach mechanism other relaxation paths such as quantum tunneling must be present, consistent with ab initio calculations and CF fitting that indicate a mixed ground state wavefunction.

$\mathbf{N a 9}\left[\mathbf{T b}\left(\mathbf{W}_{\mathbf{5}} \mathbf{O}_{18}\right)_{2}\right](\mathbf{T b})$. We have recently communicated preliminary INS studies and $a b$ initio CASSCF/RASSI calculations for $\mathbf{T} \mathbf{b},{ }^{32}$ the results of a subsequent more detailed study are reported below. The combined $\mathbf{T b}^{\mathbf{D}}$ INS spectra (Figure 5, Figure S21-S24) collected with neutron wavelengths of 2.37 and $4.74 \AA$ show two clear peaks at $1.6102(3)$ and $2.835(6) \mathrm{meV}$ (I and II) at the base temperature of $5 \mathrm{~K}$. Three more features appear at 4.99(3) $\mathrm{meV}, 6.24(2) \mathrm{meV}$, and 7.62(4) meV (Peaks III, IV, and V) upon increasing the temperature. The temperature and $\boldsymbol{Q}$ dependence (Figure S25-S26) clearly indicate that peaks I and II are CF excitations from the ground state, whereas peaks III, IV, and V are classified as hot transitions from excited CF levels based on $T$ dependence and comparison with the INS spectra of diamagnetic $\mathbf{Y}^{\mathbf{D}}$ analog (Figure S27). The peak occurring at $c a .0 .4 \mathrm{meV}$ is classified as spurious given its absence from the energy gain of the spectrum and the lack of $T$ dependence. 


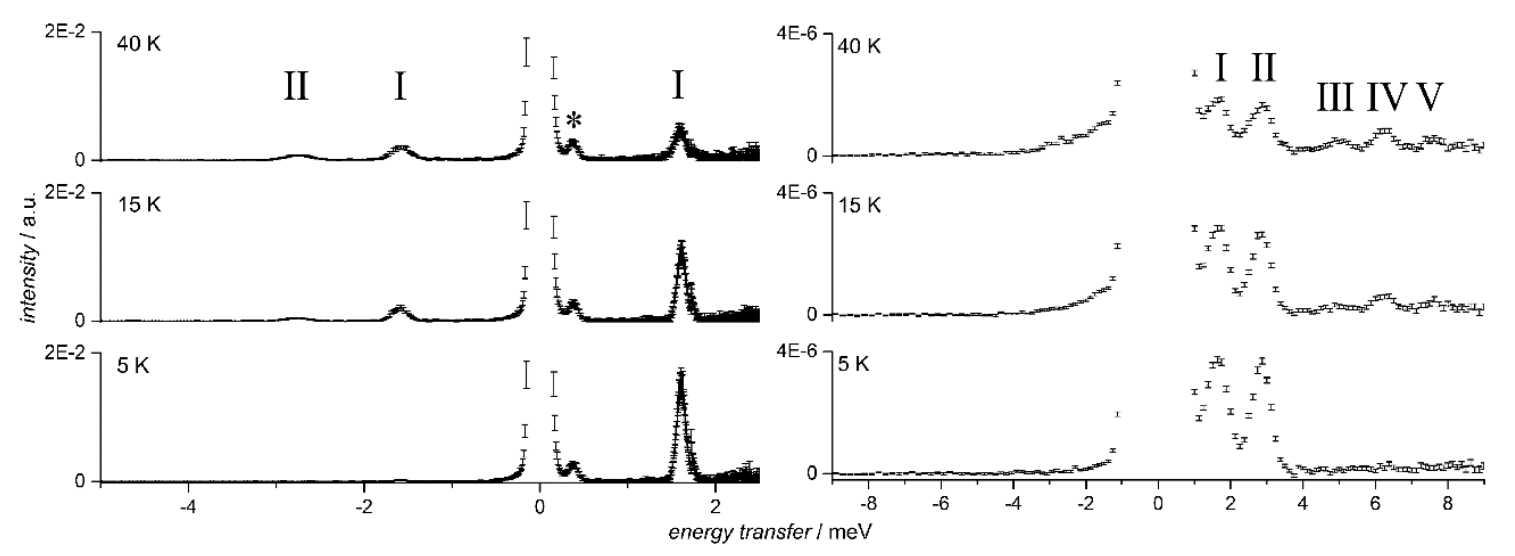

Figure 5. Variable temperature $(5,15$ and $40 \mathrm{~K})$ INS spectra of $\mathbf{T b}^{\mathbf{D}}$. Left: $4.74 \AA$ neutron wavelength spectra with integration interval $0.8 \AA^{-1}<Q<2.4 \AA^{-1}$. Right: $2.37 \AA$ neutron wavelength spectra with integration range $0.8 \AA^{-1}<Q<2.4 \AA^{-1}$ and binning interval $0.125 \mathrm{meV}$. Positive energies correspond to neutron energy loss. Magnetic transitions are labelled with Roman numerals and the spurious peak is indicated by an asterisk.

Ab initio CASSCF/RASSI calculations for $\mathbf{T b}$ are in agreement with the findings of our previous communication $^{32}$ in terms of energies and wavefunction composition. The ab initio calculations reproduce very well the temperature dependence of $\chi_{M} T$ and of the magnetization as function of magnetic field, both for the isolated polyanion and when incorporating the electrostatic effects of the crystal lattice (Figure 6, Figure S28-S29). The corresponding ab initio simulated INS spectrum (Figure 6) reproduces very well both the position and relative intensities of the peaks. The decomposition of the ab initio wavefunctions corresponding to the ground state manifold along the principal magnetic axis of the first excited pseudo-doublet (the g-tensor is not defined for the singlet ground state), which is loosely aligned with the pseudo $C_{4}$ axis of the molecule (Figure S30-S31), affords almost pure $\left| \pm M_{J}\right\rangle$ components (Table S8-S9). The 
ordering of the states obtained from the $a b$ initio calculations indicates a ground state singlet with $M_{J}=0(99 \%)$ composition, in agreement with the findings of Coronado et al. and consistent with the absence of SMM behavior. ${ }^{54}$ Because of the non-Kramers nature of $\mathrm{Tb}^{\mathrm{III}}$, the lack of rigorous axiality of the system renders all the excited doublets as pseudo-doublets. The first four excited CF levels are determined to be the non-degenerate components of two markedly split pseudo-doublets, having respectively $100 \%| \pm 1\rangle$ and $100 \%| \pm 2\rangle$ character, with ab initio calculations quantitatively accounting for the energy separation between the non-degenerate components of the first excited pseudo-doublet. The remaining excited CF levels are predicted to be quasi-degenerate, almost pure $\left| \pm M_{J}\right\rangle$ doublets (Figure 7, Table S10-S11). 

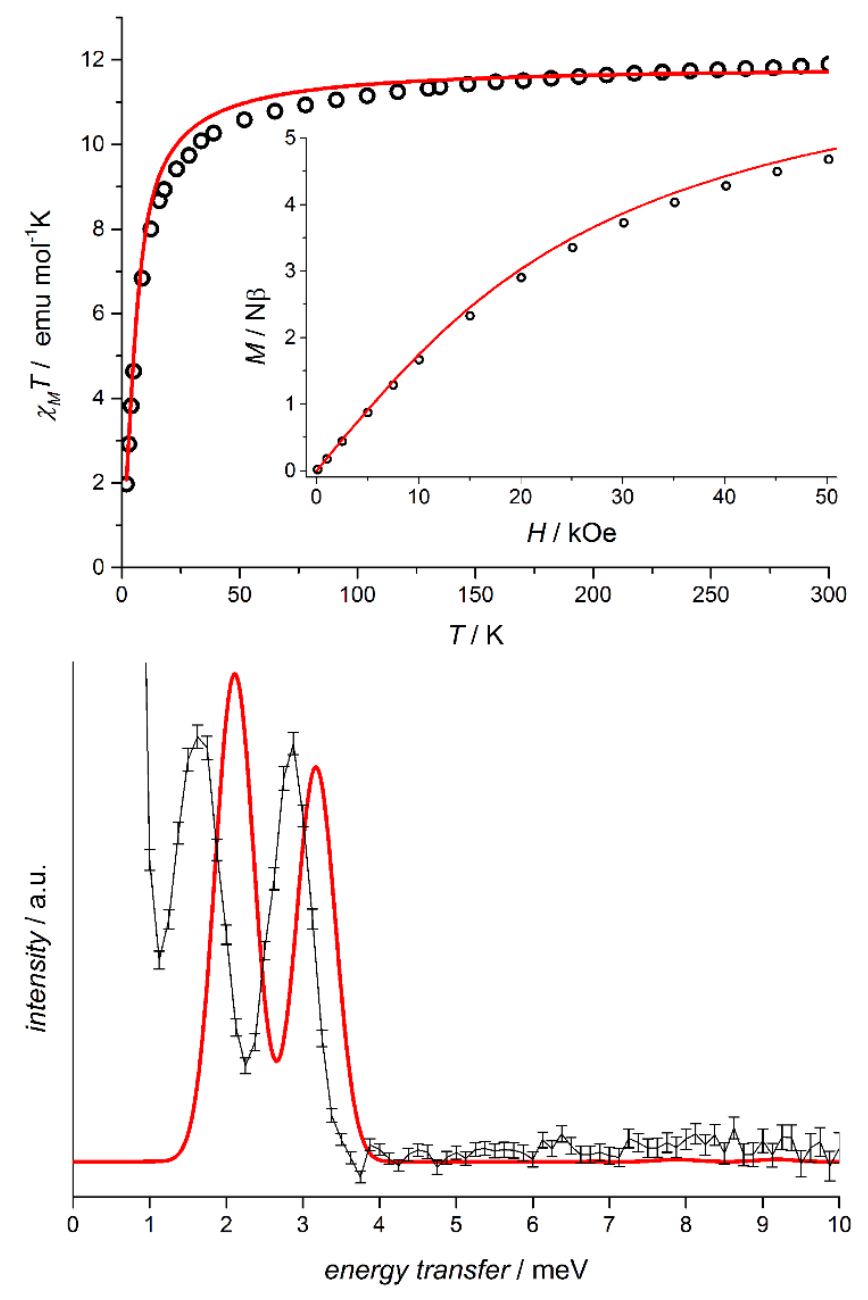

Figure 6. Top: experimental (black circles) and ab initio calculated (red line) magnetic data for $\mathbf{T b}^{\mathbf{D}}$. Main: $T$ dependence of $\chi_{M} T$ product in the $2-300 \mathrm{~K}$ range. Inset: magnetization as function of magnetic field at $2 \mathrm{~K}$. Bottom: experimental (black line) and ab initio simulated (red line) INS spectrum of $\mathbf{T} \mathbf{b}^{\mathbf{D}}$ at $5 \mathrm{~K}$. 


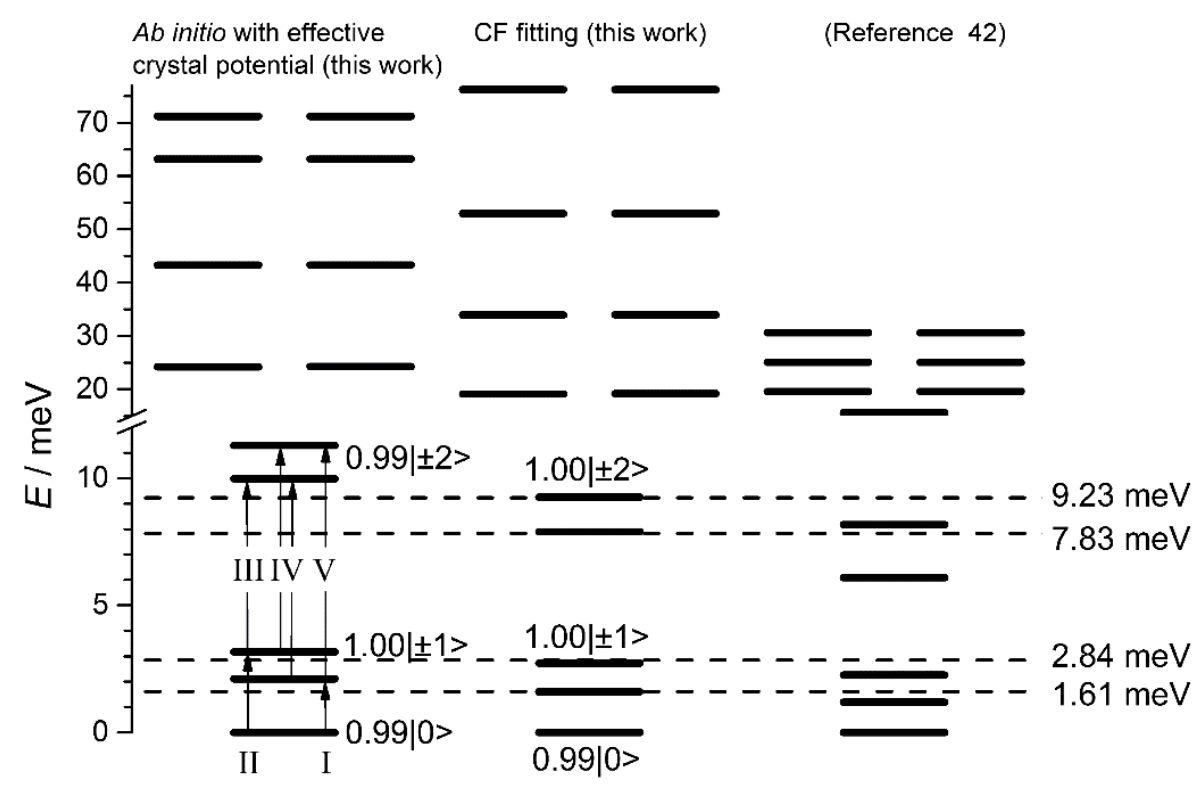

Figure 7. Energy level diagram for $\mathbf{T} \mathbf{b}^{\mathbf{D}}$ corresponding to the $J=6$ ground multiplet. Horizontal dashed lines indicate the CF levels experimentally observed with INS. Arrows correspond to the assignments of the CF transitions to the observed INS peaks, based on ab initio calculations. Comparison with energies reported by Coronado et $a l .{ }^{42}$ is provided.

In comparison with calculations for the isolated anion, the inclusion of the electrostatic potential of the crystal lattice affords a smaller overall energy range for the $J=6$ ground state manifold and better reproduction of the experimental barycenter energy for the non-degenerate components of the first excited pseudo-doublet (Table S10-S11). The energy scale of the ground state multiplet and the wavefunction composition determined by our CASSCF/RASSI calculations are similar to those obtained by the effective point charge model developed for the $\mathrm{Na} 9\left[\mathrm{Ln}\left(\mathrm{W}_{5} \mathrm{O}_{18}\right)_{2}\right]$ family by Coronado et. al, and while the former overestimate the energy of the barycenter of the pseudo-doublets, the latter underestimate it. However, ab initio calculations are 
slightly more accurate in predicting the energy gaps between the non-degenerate components of the first two pseudo-doublets (Figure 7).

On the basis of ab initio calculations and the $\Delta M_{J}= \pm 1$ INS selection rule, ${ }^{85}$ peaks I and II are assigned to the transition from the $|0\rangle$ singlet ground state to the two non-degenerate components of the first excited $| \pm 1\rangle$ pseudo-doublet (Figure 7). The electronic structure obtained from ab initio calculations also allows the assignment of peaks III, IV, and V as hot transitions between the first excited $| \pm 1\rangle$ pseudo-doublet and the second excited $| \pm 2\rangle$ pseudodoublet. The fact that despite the presence of four possible excitations only three INS peaks are observed is rationalized by the ab initio model that predicts similar energy splittings of the two pseudo-doublets. Peak III is assigned to the transition from the highest energy component of $| \pm 1\rangle$ to the lowest energy component of $| \pm 2\rangle$, while peak $V$ is assigned to the transition between the lowest energy component of $| \pm 1\rangle$ to the highest energy component of the pseudodoublet $| \pm 2\rangle$. Peak IV is the convolution of two transitions accidentally occurring at about the same energy: the transition between the lowest component of $| \pm 1\rangle$ and the lowest energy component of $| \pm 2\rangle$ and the transition from the highest component of $| \pm 1\rangle$ to the highest energy component of $\mid \pm 2$. In light of the scheme of energy levels calculated ab initio, the energies for the components of the $| \pm 1\rangle$ state are 1.61 and $2.84 \mathrm{meV}$, corresponding directly to the cold transitions associated with peaks I and II. The energies of the components of $| \pm 2\rangle$ are instead obtained as a sum of the energies of the emitting states plus the energy at which the appropriate hot transition occurs, hence $7.83 \mathrm{meV}$ and $9.23 \mathrm{meV}$ respectively.

In our previous communication, we have shown how, analogous to the above mentioned Er case, a satisfactory simulation of the energy spectrum of the low lying states of $\mathbf{T b}$ can only 
be achieved by incorporating non-axial contributions in a very simple CF Hamiltonian (Equation 4):

$$
\widehat{H}_{C F}=B_{2}^{0} \widehat{O}_{2}^{0}+B_{2}^{2} \widehat{O}_{2}^{2}+B_{4}^{4} \widehat{O}_{4}^{4}
$$

clearly demonstrating low symmetry effects in this system. ${ }^{32}$ The choice of which CF parameters to include in the truncated Hamiltonian is made on the basis of observations and symmetry arguments. Only the diagonal $B_{2}^{0} \mathrm{CF}$ parameter is needed to reproduce the barycenter of the energies of the first two excited pseudo-doublets. The inclusion of the other two diagonal CF parameters $B_{4}^{0}$ and $B_{6}^{0}$ does not improve the fitting and they are therefore omitted in order to avoid overparametrization of our simple model. The $B_{2}^{2}$ and $B_{4}^{4}$ extra-diagonal CF parameters, allow the efficient mixing of the $| \pm 1\rangle$ and $| \pm 2\rangle$ states, respectively, and are used to model the splitting of the non-degenerate components of the two first excited pseudo-doublets (Table 1). As in the case of $\mathbf{E r}$, these are not the only symmetry allowed extra-diagonal parameters in the $C_{l}$ point group of the molecule and they must be regarded as effective CF parameters summarizing all the off-diagonal contributions. The fitting of the experimental INS spectra to the simplified CF Hamiltonian allows determination of CF parameters that afford accurate reproduction of the experimental energy gaps as well as of static magnetic properties (Figures 6 and 7, S32-S33).

$\mathrm{Na} 9\left[\mathrm{Ho}\left(\mathrm{W}_{5} \mathrm{O}_{18}\right)_{2}\right]$ (Ho). Compound $\mathrm{Ho}$ shows slow relaxation of the magnetization, although a lack of clear peak maxima in the AC magnetic susceptibility data prevents the determination of an effective energy barrier to the magnetization reversal. ${ }^{54}$ This compound has also been proposed as potential spin qubit due to its long coherence times apparent from EPR studies. $^{25,49}$ The INS spectra (Figure 8, Figure S34-S37) measured for $\mathbf{H o}^{\mathbf{D}}$ with a neutron wavelength of $2.37 \AA$ show two clear signals at 5.036(3) meV (peak I) and 6.070(2) meV (peak 
II) on the energy loss side of the spectrum. On the basis of the $\boldsymbol{Q}$ and temperature dependence (Figure S38-S39), as well as by comparison with the diamagnetic $\mathbf{Y}^{\mathbf{D}}$ analog (Figure S40), these peaks are classified as $\mathrm{CF}$ transitions from the ground state. A third peak is at $c a .7 .3 \mathrm{meV}$ is assigned as spurious as it is absent in the energy gain region of the spectra and temperature independent. As a consequence of the detailed balance principle, a broad envelope corresponding to peaks I and II is evident on the energy gain side of the $2.37 \AA$ spectrum, however these peaks are poorly resolved due to the lower instrumental resolution in this region. The spectra collected with a neutron wavelength of $4.74 \AA$ (Figure 8, left) show clearly resolved peaks at $c a$. -5 and -6 meV (I and II) on the energy gain side and do not show any features on the energy loss side, indicating that no transitions occur below $2.5 \mathrm{meV}$.

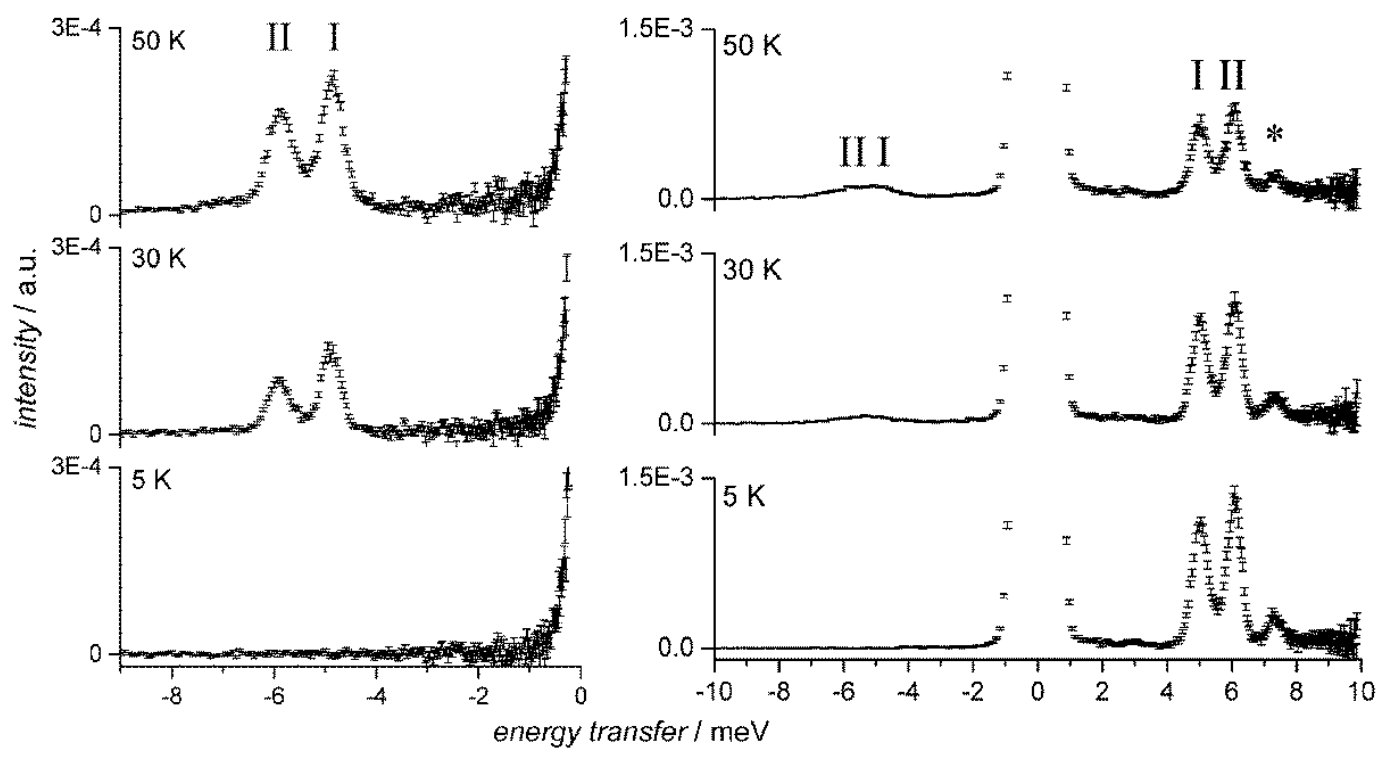

Figure 8. Variable temperature INS spectra of $\mathbf{H o}^{\mathbf{D}}$. Left: $4.74 \AA$ neutron wavelength spectra with integration interval $1.15<Q<1.65 \AA^{-1}$. Right: $2.37 \AA$ neutron wavelength spectra with integration interval $1.15<Q<1.65 \AA^{-1}$. Positive numbers correspond to neutron energy loss. 
Magnetic transitions are labelled with Roman numerals and the spurious peak is indicated by an asterisk.

$\mathrm{Ho}^{\mathrm{III}}$ ion is a non-Kramers ion and in non-rigorous axial symmetry, as occurs in $\left[\mathrm{Ho}\left(\mathrm{W}_{5} \mathrm{O}_{18}\right)_{2}\right]^{9-}$, it is reasonable to expect the removal of the degeneracy for some of the otherwise doubly degenerate microstates of the ground multiplet. ${ }^{86} \mathrm{Ab}$ initio calculations indicate (Figure 9, Table S12-S13) in fact that the ground state is a pseudo-doublet with almost degenerate components separated by $c a .0 .01 \mathrm{meV}\left(0.085 \mathrm{~cm}^{-1}\right)$. This value is qualitatively in agreement with the $0.04 \mathrm{meV}\left(0.3 \mathrm{~cm}^{-1}\right)$ value reported by EPR studies for the tunneling gap of the ground state of Ho, responsible of the long coherence times of this molecule. ${ }^{25,49}$ Very close in energy, the calculated first and second excited states are also pseudo-doublets, the components of which are separated by 0.27 and $0.17 \mathrm{meV}$ respectively. The calculated energy gap from the ground state, averaged over the two components of the lowest-lying excited pseudo-doublet, is ca. $4.0 \mathrm{meV}$, whereas the second excited pseudo-doublet is separated from the ground state by an average $c a .4 .6 \mathrm{meV}$ gap. 


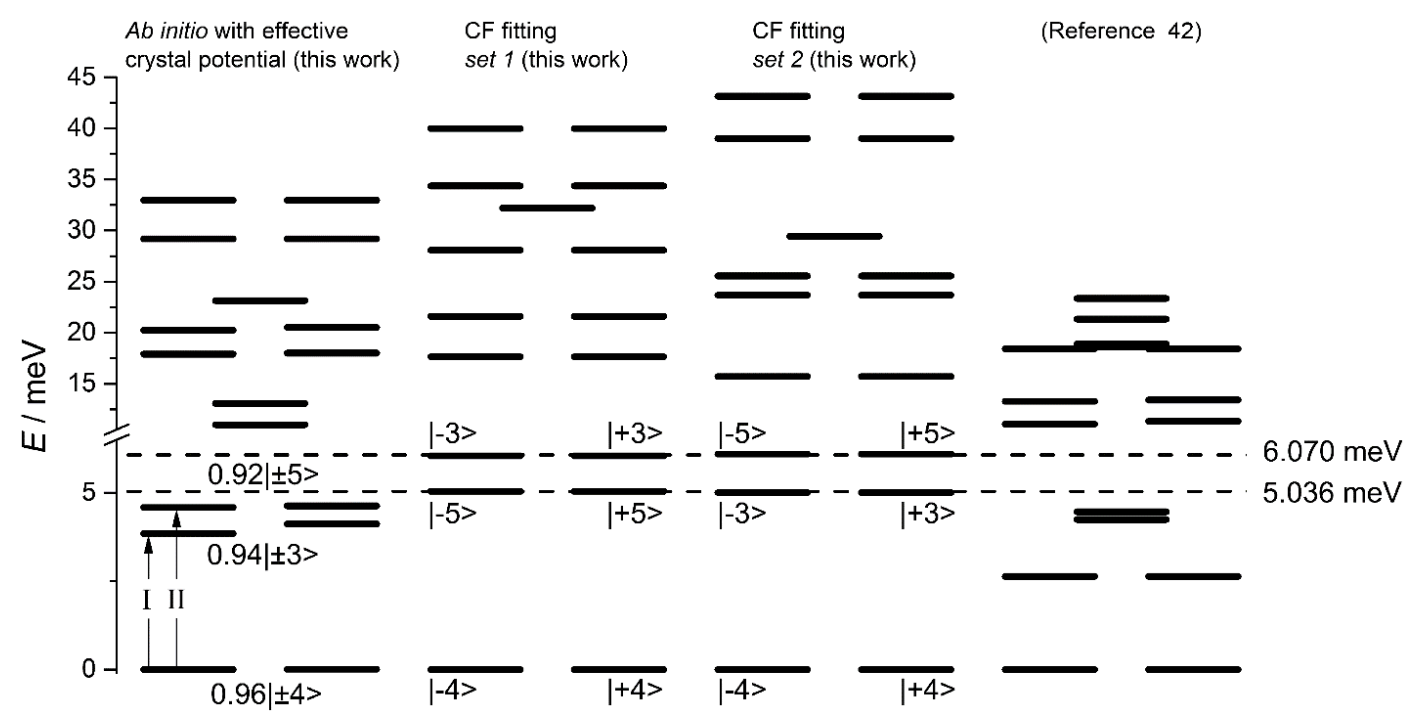

Figure 9. Energy levels diagram for $\mathbf{H o}^{\mathbf{D}}$. Horizontal dashed lines indicate the energy levels experimentally observed with INS. Arrows correspond to the assignments of the CF transitions to the observed INS peaks, based on the ab initio model (each arrow summarizes the contribution of four distinct transitions between the non-degenerate components of the pseudo-doublets, see text). Comparison with energies reported by Coronado et al. ${ }^{42}$ is provided.

Decomposition of the CASSCF/RASSI wavefunctions corresponding to the CF split ground state multiplet in a $2 J+1$ pseudo-spin basis set is extremely sensitive to the choice of the quantization axis (Table S14-S17), which has also been reported for ab initio CASSCF/RASSI calculations on $\left(\mathrm{Bu}_{4} \mathrm{~N}\right)\left[\mathrm{HoPc}_{2}\right] \cdot 2 \mathrm{DMF}$ with similar pseudo $D_{4 d}$ symmetry. ${ }^{29}$ Using the $\mathrm{z}-$ component of the $g$-tensor calculated for the ground state pseudo-doublet, which forms a $15^{\circ}$ angle with the pseudo $C_{4}$ axis (Figure S41-S42), wavefunctions that are mixed over a wide range of $M_{J}$ quantum numbers are obtained. By using the pseudo $C_{4}$ axis (Ho1-W1 direction) as quantization axis, almost pure $M_{J}$ components are obtained for each microstate. The predicted admixture of the ground state is consistent with the fast relaxation of the magnetization observed 
which prevents the appearance of clear peaks in out-of-phase ac susceptibility measurements, suggesting that quantum tunneling is the dominant relaxation mechanism. Ab initio calculations reproduce accurately the low $T$ value of $\chi_{M} T$ product and the magnetization curve at $2 \mathrm{~K}$, indicating that wavefunction corresponding to the ground state is correctly described (Figure 10, Figure S43-S44).
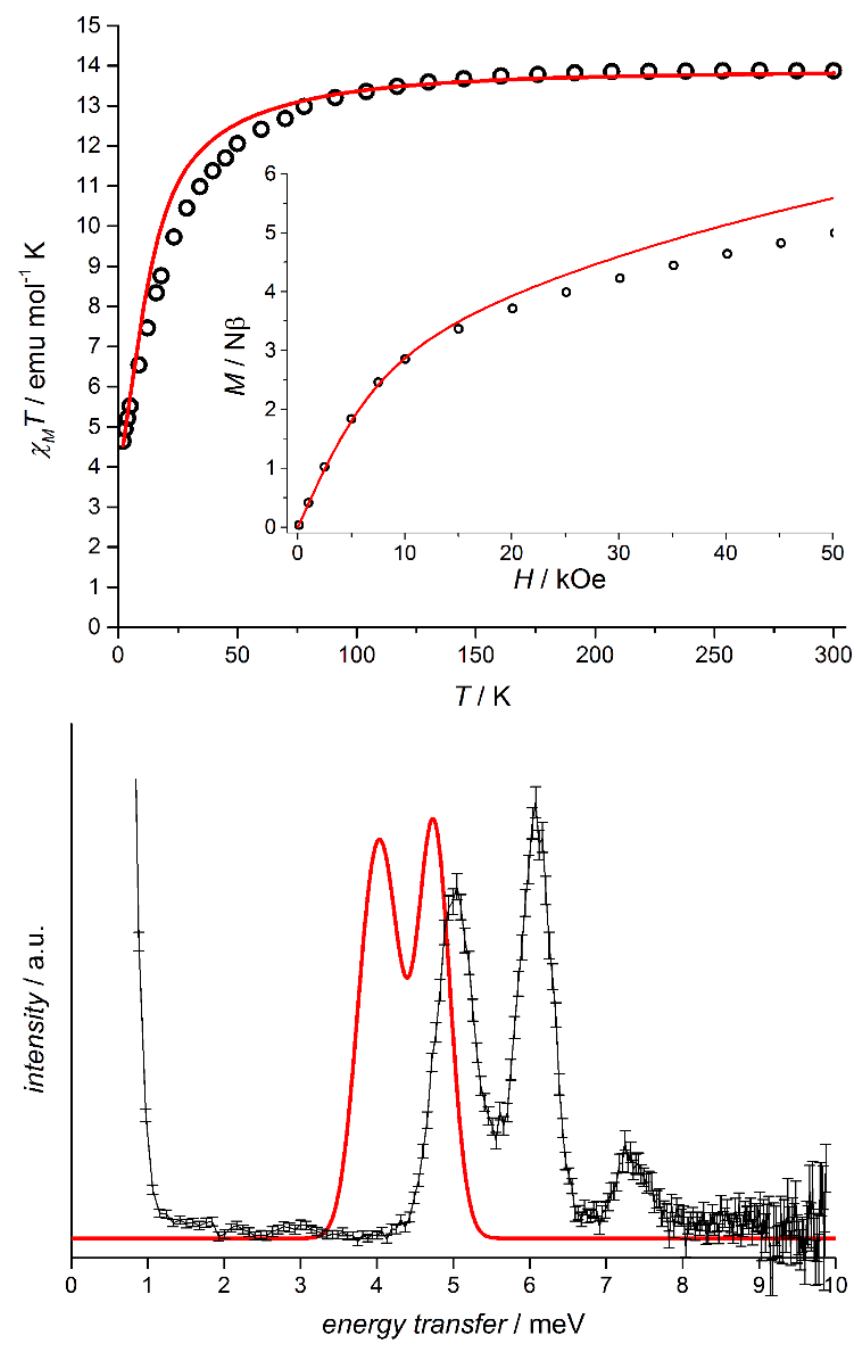

Figure 10. Top: experimental (black circles) and ab initio calculated (red line) magnetic data for $\mathbf{H o}^{\mathrm{D}}$. Main: $T$ dependence of $\chi_{M} T$ product in the $2-300 \mathrm{~K}$ range. Inset: magnetization as function 
of magnetic field at $2 \mathrm{~K}$. Bottom: Experimental (black) and $a b$ initio simulated (orange) INS spectrum of $\mathbf{H o}^{\mathbf{D}}$ at $5 \mathrm{~K}$.

The energy spectrum calculated ab initio is consistent with the transitions observed by INS. Moreover the matrix elements of the magnetic moment calculated between the RASSI states corresponding to the $J=8$ ground state multiplet for Ho show that the only INS active transitions at $5 \mathrm{~K}$ are those between the ground state and first and second excited pseudodoublets. The ab initio simulated INS spectrum (Figure 10) reproduces well both position and relative intensity of the experimental INS peaks. Peaks I and II can both then be rationalized as the convolution of the four signals from the quasi doubly degenerate ground state of $\mathbf{H o}$ to the quasi doubly degenerate first and second excited states. Because of the resolution of the instrument at $2.37 \AA$ neutron wavelength ( $c a .850 \mu \mathrm{eV}$ at the elastic line) the calculated four separate components of each INS peaks are not resolved and the actual spectrum consists of two peaks (Figure 10). The ab initio results, when compared to the experimental INS data, tend to underestimate the energy gaps from the ground state to the excited levels (Figure 9). The calculations that include the effect of the crystal lattice afford a slightly larger energy range for the ground state manifold and are in better agreement with the experimental energy gaps in comparison to calculations on the isolated anion (Table S12-S13). The effective point charge model proposed by Coronado et al ${ }^{42}$ underestimates the energy scale of the ground state multiplet and reproduces the energy gap between the ground state and the first two excited pseudo-doublets (Figure 9) less well than our ab initio results. 
Interestingly the low symmetry effects evident for $\mathbf{E r}$ and $\mathbf{T b}$ are not immediately apparent from the INS transitions observed for $\mathbf{H o}^{\mathbf{D}}$. A CF Hamiltonian containing only the diagonal $B_{2}^{0}, B_{4}^{0}$, and $B_{6}^{0} \mathrm{CF}$ parameters (Equation 2) is adequate to fit the INS spectrum and the inclusion of the $B_{4}^{4}$ term, necessary to model the EPR data ${ }^{25,49}$, is not required to fit our INS spectra. This reflects the fact that the pseudo-doublets responsible for the observed INS signals are only minimally split by the extra diagonal terms of the CF Hamiltonian allowed in $C_{1}$ symmetry, and the instrumental resolution of the PELICAN spectrometer does not allow resolution of these quasi-degenerate components. The eigenvectors with definite $M_{J}$ projections of the total angular momentum that are obtained from fitting the simplified CF Hamiltonian to the experimental INS data have to be considered as approximations of the real wavefunctions. Two possible sets of CF parameters have been determined (Table 1) together with the corresponding energy levels (Figure 9). Both sets predict the same $\mid \pm 4>$ ground state, but differ for the ordering of the excited levels. Set 1 predicts as first and second excited doublet the $\mid \pm 5>$ and $| \pm 3\rangle$ state respectively, whereas set 2 predicts $| \pm 3\rangle$ and $| \pm 5\rangle$ as first and second excited states respectively (Figure 9). The two sets predict equally well the position of the two observed cold INS transitions, but whereas set 1 affords an excellent simulation of the relative intensity of the two INS peaks (Figure S45), the simulation achieved with set 2 is poorer. Both sets reproduce equally well the $T$ dependence of $\chi_{M} T$ product in the $2-300 \mathrm{~K}$ range as well as the magnetization curve at $2 \mathrm{~K}$, with no appreciable difference in the quality of fitting (Figure S46S47). To observe the effects of the low symmetry of the system it would be necessary to access higher energy transitions for which, as predicted by ab initio calculations (Figure 9), the splitting of quasi-degenerate components would be within the resolution of PELICAN using $2.37 \AA$ 
wavelength neutrons. Unfortunately, these levels are not accessible within the maximum energy transfer of the PELICAN spectrometer in the adopted configuration.

$\mathbf{N a 9}\left[\mathbf{N d}\left(\mathbf{W}_{5} \mathrm{O}_{18}\right)_{2}\right](\mathbf{N d})$. Compound $\mathbf{N d}$ is a SMM in applied magnetic field (1 kOe) with an experimental effective energy barrier to magnetization reversal of $6.4 \mathrm{meV} .{ }^{42}$ The INS spectra of $\mathbf{N d}^{\mathbf{D}}$ (Figure 11, Figure S48-S51) exhibit a single magnetic peak (peak I) at $10.0 \mathrm{meV}$ in the energy loss region of the $5 \mathrm{~K}$ spectrum with a neutron wavelength of $2.37 \AA$. This peak is classified as a magnetic transition from the ground state, based on the $T$ dependence and $\boldsymbol{Q}$ dependence (Figure S52). A peak at $2.7 \mathrm{meV}$ visible at $5 \mathrm{~K}$, together with features in the 5-9 meV region, are all classified as phonons based on the $T$ dependence and $S(\boldsymbol{Q}, \omega)$ diagram. The assignment of phonons for $\mathbf{N d}^{\mathbf{D}}$ is complicated by the fact that in contrast to $\mathbf{E} \mathbf{r}^{\mathbf{D}}, \mathbf{T b}^{\mathbf{D}}$, and $\mathbf{H o}^{\mathbf{D}}$ it was not possible to obtain an isomorphous diamagnetic $\mathrm{Y}^{\mathrm{III}}$ analog. Peak I is still visible in the $100 \mathrm{~K}$ INS spectrum collected with neutron wavelength $2.37 \AA$ and disappears at $200 \mathrm{~K}$. The temperature dependence of the intensity peak I is consistent with the large energy gap between the ground state and the first excited CF level associated with this cold transition (Figure S53). 


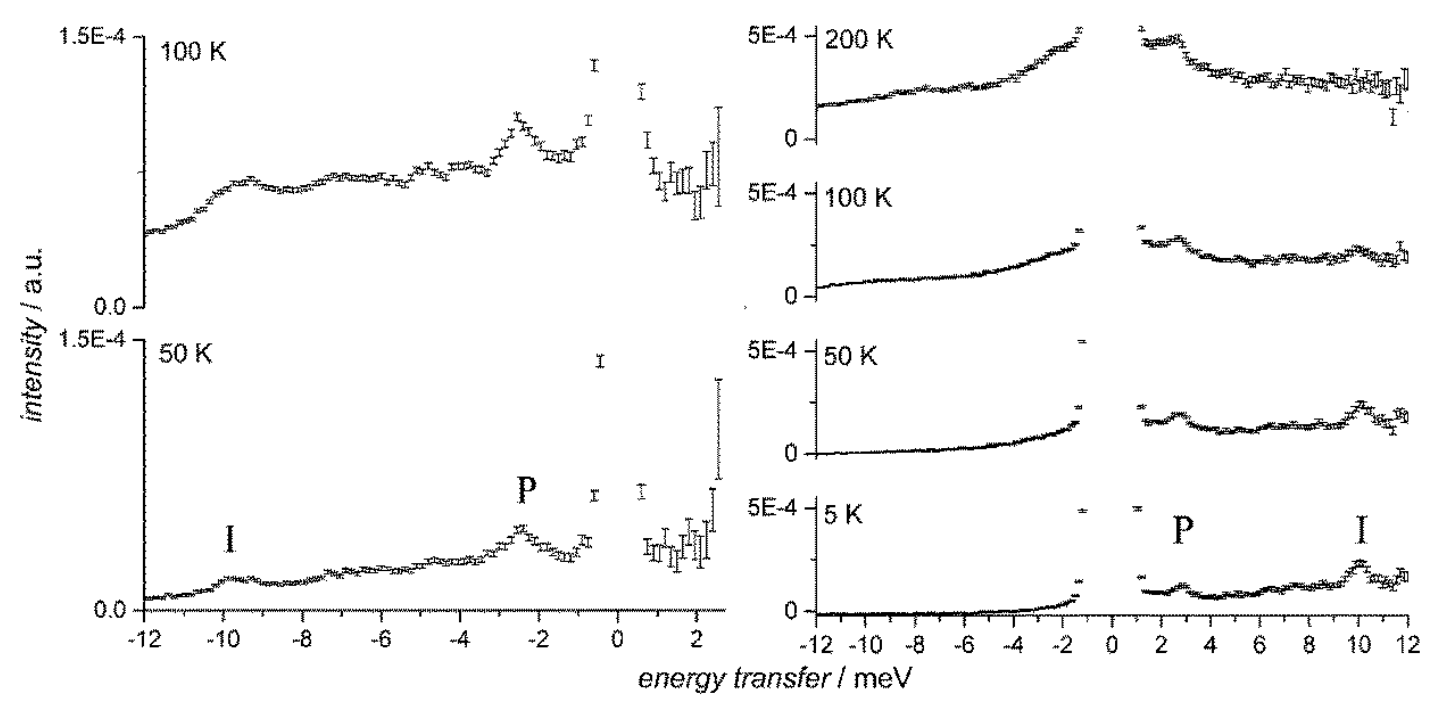

Figure 11. Variable temperature INS spectra of $\mathbf{N d}^{\mathbf{D}}$. Left: $4.74 \AA$ A neutron wavelength spectrum with integration interval over all $Q$ range and binning interval $0.15 \mathrm{meV}$. Right: $2.37 \AA$ neutron wavelength spectrum with integration interval over all $Q$ range and binning interval $0.15 \mathrm{meV}$. Positive numbers correspond to neutron energy loss. The magnetic transition is labelled with Roman numerals and the phonon signal is designated as " $\mathrm{P}$ ".

Independent $a b$ initio calculations were carried out on the two crystallographically distinct moieties containing $\mathrm{Nd} 1$ and Nd1'. The two resulting energy patterns (Figure 12, Table S18-S21) corresponding to the ground state multiplet are similar, with a slightly larger energy scale for $\mathrm{Nd1}$. Calculation of the matrix elements of the magnetic moment between the microstates of the ground multiplet indicates that the only INS active transition at $5 \mathrm{~K}$ is from the ground state to the first excited $\mathrm{KD}$ (Figure 12) for both the $\mathrm{Nd}^{\mathrm{III}}$ ions, allowing the assignment of peak I to this cold transition. The energy of the INS transition is predicted to be almost identical for the two $\mathrm{Nd}^{\mathrm{III}}$ centers, agreeing extremely well with the single magnetic peak evident in the INS spectra. The simulation of static magnetic properties does not show significant differences between the 
two $\mathrm{Nd}^{\mathrm{III}}$ centers and the experimental curves are very well reproduced (Figure 13, Figure S54S55) using the average contribution of the signals of the two inequivalent $\mathrm{Nd}^{\mathrm{III}}$ ions.

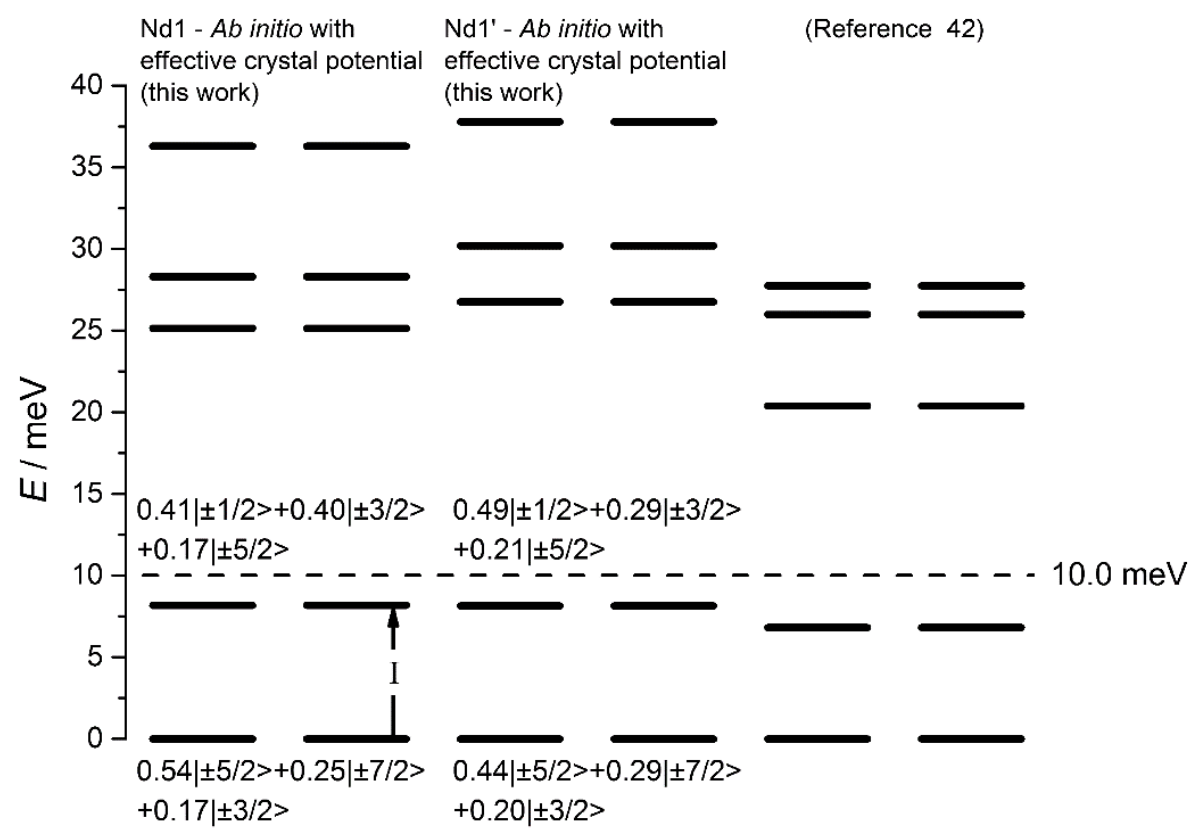

Figure 12. Energy level diagram for $\mathbf{N d}^{\mathbf{D}}$ calculated for the two crystallographically distinct moieties containing $\mathrm{Nd} 1$ and $\mathrm{Nd}^{\prime}$, respectively. Horizontal dashed line indicates the energy level experimentally observed with INS. The arrow corresponds to the assignment of the CF transition to the observed INS peak, based on the $a b$ initio model. Comparison with energies reported by Coronado et al. $^{42}$ is provided. 

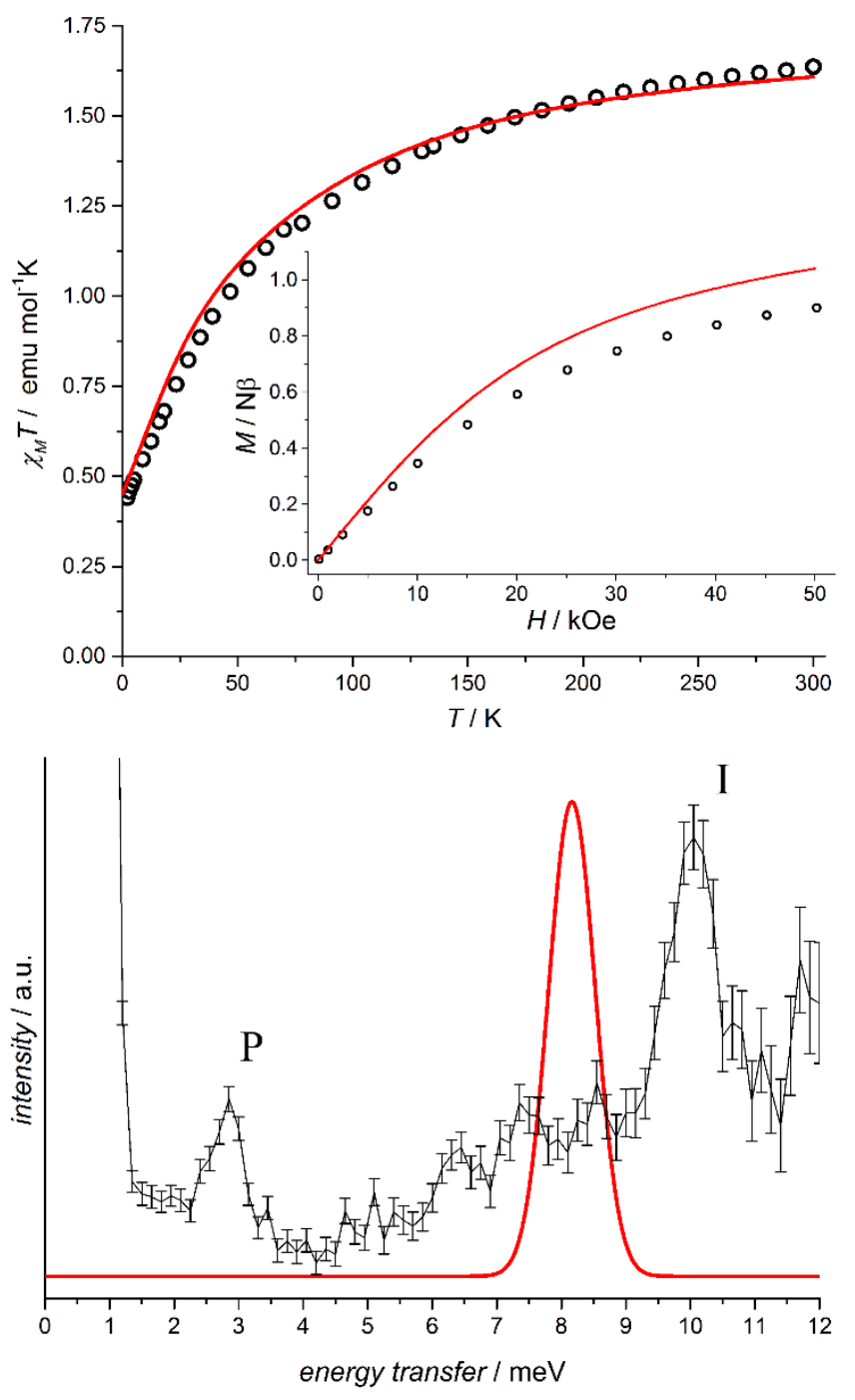

Figure 13. Top: experimental (black circles) and ab initio calculated (red line) magnetic data for $\mathbf{N d}^{\mathbf{D}}$ as averaged signals of $\mathrm{Nd} 1$ and $\mathrm{Nd} 1^{\prime}$. Main: $T$ dependence of $\chi_{M} T$ product in the 2-300 $\mathrm{K}$ range. Inset: magnetization as function of magnetic field at $2 \mathrm{~K}$. Bottom: experimental (black line) and ab initio simulated (red line) INS spectrum of $\mathbf{N d}^{\mathbf{D}}$ at $5 \mathrm{~K}$ as average contribution of $\mathrm{Nd} 1$ and $\mathrm{Nd} 1$ ' signals.

The decomposition of the ab initio wavefunction was performed using as quantization axis the principal magnetic axis of the ground state doublet (Figure S56-S59). For both Nd1 and $\mathrm{Nd1}$ ' the calculations on the isolated polyanion and those including the fractional point charges 
distribution indicate an extremely mixed ground and first excited state (Table S22-S25), the former with main components $| \pm 3 / 2\rangle,| \pm 5 / 2\rangle$, and $| \pm 7 / 2\rangle$, while the latter with main components $| \pm 1 / 2\rangle,| \pm 3 / 2\rangle$, and $| \pm 5 / 2\rangle$. As in the case of $\mathbf{H o}$, the ab initio calculations underestimate the energy gap between the ground and first excited states, although the introduction of distribution of point charges into the calculations improves this for both $\mathrm{Nd} 1$ and $\mathrm{Nd}^{\prime}$. The first energy gap reported by Coronado et al. is even less than that predicted ab initio. Interestingly the energy gap between the ground state and the first excited state $(10.0 \mathrm{meV})$ is higher than the effective energy barrier $U_{\text {eff }}(c a .6 .4 \mathrm{meV})$ reported for the relaxation process of another isostructural crystalline phase of $\mathbf{N d}{ }^{42}$ indicating that relaxation paths other than the proposed relaxation via the first excited state must be present, consistently with the extremely mixed ground state predicted by ab initio calculations and the necessity of applying an external magnetic field to suppress quantum tunneling to observe slow relaxation of the magnetization. The observation of only one magnetic peak in our INS spectra precludes any attempt to independently fit the INS spectra to a CF model.

$\operatorname{Na} 9\left[\mathbf{D y}\left(\mathbf{W}_{5} \mathbf{O}_{18}\right)_{2}\right]$ (Dy). INS spectra were not collected for Dy because of the high neutron absorption cross-section of Dy and W (994 and 18.3 barn respectively at $1.8 \AA$ ). ${ }^{87} \mathrm{We}$ did however perform CASSCF/RASSI calculations, which on the isolated $\left[\mathrm{Dy}\left(\mathrm{W}_{5} \mathrm{O}_{18}\right)_{2}\right]^{9-}$ anion, indicate a ground state with predominant character $| \pm 15 / 2\rangle$ (81\%) with further contamination from $| \pm 11 / 2\rangle(8 \%)$ and $| \pm 7 / 2\rangle$ (4\%). Incorporation of the electrostatic potential of the crystal lattice affords a more mixed ground state, although still with a predominant $| \pm 15 / 2\rangle$ character (56\%), with contributions from $| \pm 11 / 2\rangle$ (22\%) and $| \pm 7 / 2\rangle$ (8\%) (Table S26-S27). The introduction of the crystal lattice also stabilizes the calculated energy levels of the excited KDs 
with respect to the ground state, with the largest stabilization being $c a .30 \%$ for the first excited KD (Figure 14, Table S28-S29).

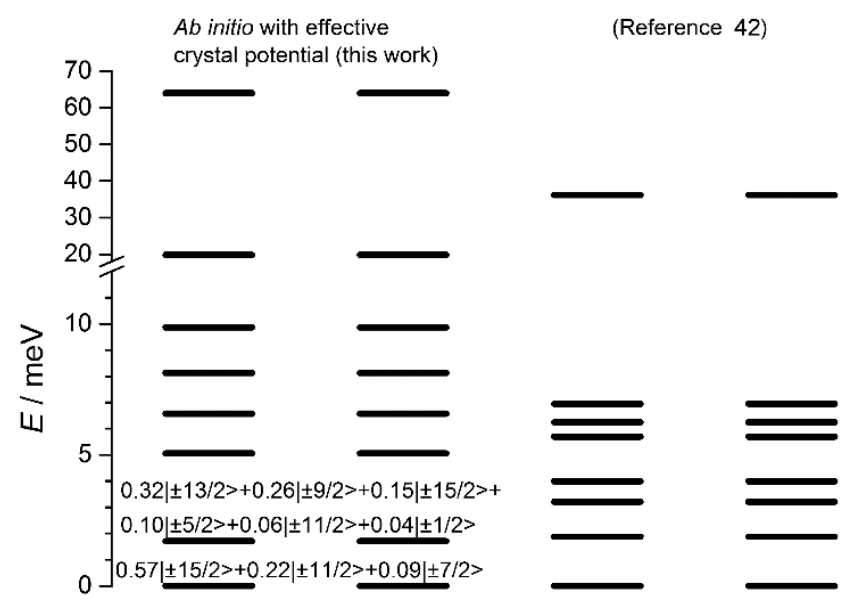

Figure 14. Energy level diagram for $\mathbf{D y}$ for the wavefunctions corresponding to the ground state multiplet $J=15 / 2$. Comparison with energies reported by Coronado et al. is provided. ${ }^{42}$

We simulated the magnetic properties (Figure 15, Figure S60-S61) of Dy as well as the theoretical INS spectrum (Figure S62). The reproduction of the $\chi_{M} T$ and magnetization data significantly improves by including the crystal lattice electrostatic potential in the ab initio calculations (Figure 15, Figure S60-S61). This effect is evident in the description of both the low $T$ limit of $\chi_{M} T$ and in the $2 \mathrm{~K}$ magnetization curve, indicating a better description of the ground state wavefunction. Interestingly, the ab initio calculated $g$-tensor of the ground state is oriented in a direction perpendicular to the pseudo $C_{4}$ axis (Figure S63-S64). This result is in excellent agreement with the purely electrostatic model developed for $\mathrm{Dy}^{\mathrm{III}}$ complexes implemented in the software MAGELLAN (Figure S65). ${ }^{35}$ Although experimental confirmation is still required, it 
strongly suggests that, as found for Ln-SMMs, even in the presence of high pseudo-symmetry, the anisotropy axis is not necessarily aligned to the pseudo-symmetry axis. ${ }^{35,88,89}$ According to the oblate versus prolate description, ${ }^{3,35}$ in the oversimplified model of an axial $D_{4 d}$ local symmetry of the $\left[\mathrm{Dy}\left(\mathrm{W}_{5} \mathrm{O}_{18}\right)_{2}\right]^{9-}$ anion, the equatorial ${ }^{46,54}$ arrangement of the POM O-based negative charges would stabilize the prolate Dy ${ }^{\mathrm{III}}$ charge distribution that characterizes the projections of low total angular momentum, with the anisotropy axis strictly aligned to the $C_{4}$ molecular axis (Figure 16 left). The reality appears to be more complicated and the calculations suggest that the distorted $D_{4 d}$ local symmetry of the Dy ${ }^{\mathrm{III}}$ ion makes it more energetically favorable to accommodate an oblate charge distribution of Dy ${ }^{\text {III }} 4 f$ electrons with high total angular momentum projections, although with the anisotropy axis oriented perpendicularly to the pseudo $C_{4}$ axis (Figure 16 right).

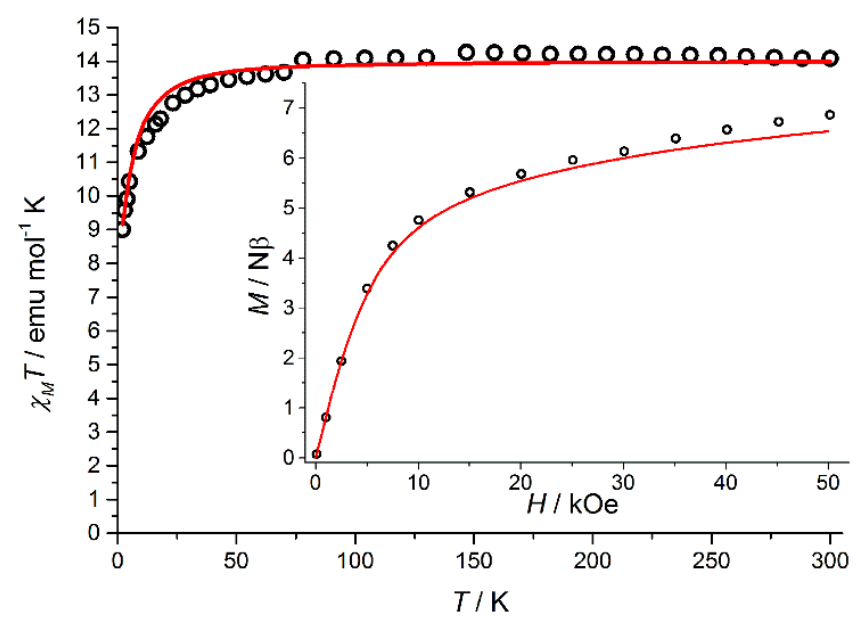

Figure 15. Experimental (black circles) and ab initio calculated (red line) magnetic data for Dy. Main: $T$ dependence of $\chi_{M} T$ product in the $2-300 \mathrm{~K}$ range with magnetic fields of $0.1 \mathrm{~T}(2-70 \mathrm{~K})$ and $1 \mathrm{~T}(70-300 \mathrm{~K})$; at $70 \mathrm{~K}$ is visible an artifact due to the switching of magnetic field. Inset: Magnetization as function of magnetic field at $2 \mathrm{~K}$. 


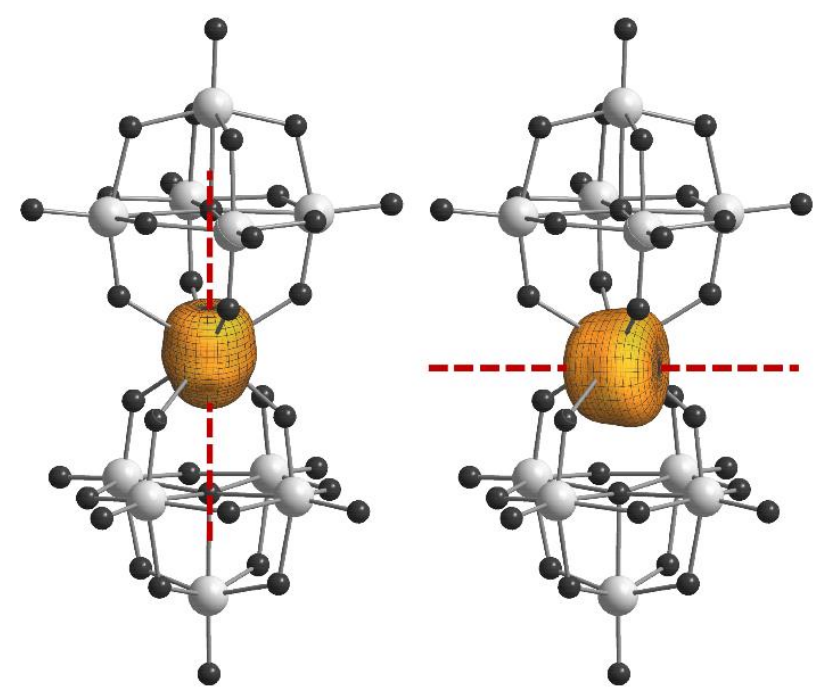

Figure 16. Dy ${ }^{I I I}$ free ion charge density distribution corresponding to different $M_{J}$ states accommodated in the POM framework. Left: prolate charge density distribution corresponding to $M_{J}= \pm 1 / 2$ with anisotropy axis (red dashed line) oriented along the pseudo $C_{4}$ axis of the molecule. Right: oblate charge density distribution corresponding to $M_{J}= \pm 15 / 2$ with anisotropy axis (red dashed line) oriented perpendicularly to the pseudo $C_{4}$ axis of the molecule.

In the CASSCF/RASSI calculations, as well as with the electrostatic model implemented with MAGELLAN, the orientation of the anisotropy axis is almost the same for both the isolated anion and in the presence of the effects of the crystal lattice, indicating that the origin of the anisotropy is due to the interactions within the first coordination sphere of the Dy ${ }^{\mathrm{III}}$ ion, i.e. with the coordinated $\mathrm{O}$ donor atoms of the POM ligands. The crystal electrostatic potential modelled by the use of point charges has, instead, the effect of lowering the energies of the excited states and increasing the admixture of the wavefunction compositions by introducing transverse components of the anisotropy. 


\section{Concluding Remarks}

We have carried out INS measurements on the Nd, Tb, Ho, Er analogs of the lanthanoidpolyoxometalate single-molecule magnet family $\mathrm{Na} 9\left[\mathrm{Ln}\left(\mathrm{W}_{5} \mathrm{O}_{18}\right)_{2}\right]$ and observed $\mathrm{CF}$ excitations within the $\mathrm{Ln}^{\mathrm{III}}$ ground state multiplet for all compounds. By directly accessing the experimental CF energies we were able to benchmark the ab initio CASSCF/RASSI calculations that were performed on these systems and extended also to the Dy ${ }^{\mathrm{III}}$ analog. Often mentioned for LnSMMs are discrepancies between the effective energy barrier to magnetization reversal (calculated assuming an Arrhenius energy-activated process) and the energy separations between the indirectly determined ground and first excited microstates of the relevant CF split ground multiplet. Our study has afforded direct spectroscopic evidence of such a discrepancy for Er and Nd, suggesting that even more detailed models, validated by spectroscopic measurements, are needed to accurately describe the relaxation of magnetization in these, and other, Ln-SMMs.

Low symmetry effects due to deviation from the idealized $D_{4 d}$ local symmetry of the $\mathrm{Ln}^{\mathrm{III}}$ ions are clearly evident in the INS spectra of both Kramers and non-Kramers ions such as $\mathbf{E r}^{\mathbf{D}}$ and $\mathbf{T b}^{\mathbf{D}}$. These observations make it very clear that great care must be taken when employing models that assume high local symmetry. For $\mathbf{H o}^{\mathbf{D}}$, although predicted by CASSCF/RASSI calculations for high energy CF levels of the ground state manifold, the low symmetry effects were not detected due to the limited resolution and energy range available with the INS spectrometer. As another consequence of the lack of axial symmetry, the magnetic anisotropy axis of Dy was calculated to be perpendicular to the pseudo symmetry $C_{4}$ axis, which is counterintuitive when considering the pseudo axial symmetry of the molecule. 
The inclusion of electrostatic effects of the crystal lattice on the $\mathrm{Ln}^{\mathrm{III}}$ ion in the CASSCF/RASSI calculations affords a substantially better reproduction of the experimental CF energy levels and of the simulated static magnetic properties than those obtained when calculating only the isolated $\left[\mathrm{Ln}\left(\mathrm{W}_{5} \mathrm{O}_{18}\right)_{2}\right]^{9-}$ polyanion. For Dy and Er the inclusion of the crystal lattice induces a significant change in the ground state wavefunction composition, suggesting that an opportune choice of cation or crystal packing might be exploited to tuning the magnetic properties of these molecules. We also speculate that such an approach could be used to tune the tunneling gap that renders Ho such an appealing system as qubit for quantum computing.

Finally, with Er the only exception, very good agreement between the observed and calculated spectroscopic and magnetic properties demonstrates that the ab initio CASSCF/RASSI method is a reliable and computationally affordable theoretical approach for describing the electronic and magnetic properties of the $\mathrm{Na}_{9}\left[\mathrm{Ln}\left(\mathrm{W}_{5} \mathrm{O}_{18}\right)_{2}\right]$ family. Considering the approximations that were introduced to overcome the computational challenges posed by CASSCF/RASSI calculations in presence of many tungsten atoms, our results reveal very good agreement between the energy levels calculated with CASSCF/RASSI and the lowest CF levels experimentally determined with INS, with an accuracy comparable to that found for computationally less demanding Ln complexes with organic ligands. With the exception of $\mathbf{T b}$, our CASSCF/RASSI calculations tend to underestimate the energy of the first excited levels determined experimentally, nonetheless they offer a valuable improvement for the $\mathrm{Na} 9\left[\mathrm{Ln}\left(\mathrm{W}_{5} \mathrm{O}_{18}\right)_{2}\right]$ family over current state-of-the-art effective point charge models from the literature. Importantly, as they are based on first principles, CASSCF/RASSI methods provide a 
description of the $\mathrm{Ln}^{\mathrm{III}}$ electronic structure determined by the underlying physics of these systems, rather than relying on fitting experimentally measured quantities.

\section{ASSOCIATED CONTENT}

Supporting Information. X-ray crystallographic files in CIF format, additional structural, TGA, IR, magnetic INS and ab initio data; this material is available free of charge via the Internet at http://pubs.acs.org.

\section{AUTHOR INFORMATION}

\section{Corresponding Authors}

*c.boskovic@unimelb.edu.au, asoncini@unimelb.edu.au and richardm@ansto.gov.au

\section{Author Contributions}

The manuscript was written through contributions of all authors. All authors have given approval to the final version of the manuscript.

\section{Acknowledgments}

We thank the Australian Research Council for financial support to C.B., K.S.M. and A.S. We thank the Bragg Institute, ANSTO, for access to neutron scattering facilities and for providing technical support for the INS measurements, in particular Dr K. Rule for preliminary INS 
measurements on TAIPAN triple axis spectrometer. We thank the staff of National Deuteration Facility, ANSTO, in particular Dr T. Darwish and Dr A. Leung, for useful suggestions and technical support in the deuteration of specimens for INS. M.V. and M.J.G thank AINSE for a Postgraduate Research Award and an Honours Scholarship, respectively. W.V.d.H thanks the University of Melbourne for a McKenzie Postdoctoral Fellowship. M.V thanks Dr C. Ritchie for useful discussions about polyoxometalate chemistry. Ab initio calculations were supported by use of the University of Melbourne node of NeCTAR Research Cloud computing infrastructure, a collaborative Australian research platform supported by the National Collaborative Research Infrastructure Strategy.

\section{REFERENCES}

(1) Gatteschi, D.; Sessoli, R. Quantum Tunneling of Magnetization and Related Phenomena in Molecular Materials. Angew. Chem. Int. Ed. Engl. 2003, 42, 268-297.

(2) Sorace, L.; Benelli, C.; Gatteschi, D. Lanthanides in Molecular Magnetism: Old Tools in a New Field. Chem. Soc. Rev. 2011, 40, 3092-3104.

(3) Rinehart, J. D.; Long, J. R. Exploiting Single-Ion Anisotropy in the Design of f-Element Single-Molecule Magnets. Chem. Sci. 2011, 2, 2078-2085.

(4) Luzon, J.; Sessoli, R. Lanthanides in Molecular Magnetism: So Fascinating, so Challenging. Dalton Trans. 2012, 41, 13556-13567.

(5) Woodruff, D. N.; Winpenny, R. E. P.; Layfield, R. A. Lanthanide Single-Molecule Magnets. Chem. Rev. 2013, 113, 5110-5148.

(6) Feltham, H. L. C.; Brooker, S. Review of Purely 4f and Mixed-Metal Nd-4f SingleMolecule Magnets Containing Only One Lanthanide Ion. Coord. Chem. Rev. 2014, 276, 
$1-33$.

(7) Zhang, P.; Zhang, L.; Tang, J. Lanthanide Single Molecule Magnets: Progress and Perspective. Dalton Trans. 2015, 44, 3923-3929.

(8) Liddle, S. T.; van Slageren, J. Improving f-Element Single Molecule Magnets. Chem. Soc. Rev. 2015, 44, 6655-6669.

(9) Rinehart, J. D.; Fang, M.; Evans, W. J.; Long, J. R. A N ${ }^{3-}$ Radical-Bridged Terbium Complex Exhibiting Magnetic Hysteresis at 14 K. J. Am. Chem. Soc. 2011, 133, 1423614239.

(10) Gupta, S. K.; Rajeshkumar, T.; Rajaraman, G.; Murugavel, R. An Air-Stable Dy(III) Single-Ion Magnet with High Anisotropy Barrier and Blocking Temperature. Chem. Sci. 2016, 7, 5181-5191.

(11) Liu, J.-L. J.; Chen, Y.-C.; Liu, J.-L. J.; Vieru, V.; Ungur, L.; Jia, J.-H.; Chibotaru, L. F.; Lan, Y.; Wernsdorfer, W.; Gao, S.; Chen, X.-M.; Tong, M.-L.; Gao, II Song; Chen, X.-M.; Tong, M.-L. A Stable Pentagonal Bipyramidal Dy(III) Single-Ion Magnet with a Record Magnetization Reversal Barrier over 1000 K. J. Am. Chem. Soc. 2016, 138, 5441-5450.

(12) Ungur, L.; Chibotaru, L. F. Strategies toward High-Temperature Lanthanide-Based Single-Molecule Magnets. Inorg. Chem. 2016, doi: acs.inorgchem.6b01353.

(13) Leuenberger, M. N.; Loss, D. Quantum Computing in Molecular Magnets. Nature 2001, 410, 789-793.

(14) Stepanenko, D.; Trif, M.; Loss, D. Quantum Computing with Molecular Magnets. Inorg, Chim. Acta 2008, 361, 3740-3745.

(15) Affronte, M.; Troiani, F.; Ghirri, A.; Candini, A.; Evangelisti, M.; Corradini, V.; Carretta, S.; Santini, P.; Amoretti, G.; Tuna, F.; Timco, G.; Winpenny, R. E. P. Single 
Molecule Magnets for Quantum Computation. J. Phys. D. Appl. Phys. 2007, 40, 29993004.

(16)

Dei, A.; Gatteschi, D. Molecular (Nano) Magnets as Test Grounds of Quantum Mechanics. Angew. Chem. Int. Ed. Engl. 2011, 50, 11852-11858.

(17) Éthier-Majcher, G.; St-Jean, P.; Boso, G.; Tosi, A.; Klem, J. F.; Francoeur, S. Complete Quantum Control of Exciton Qubits Bound to Isoelectronic Centres. Nature Commun. 2014, 5, 3980 .

(18) Fataftah, M. S.; Zadrozny, J. M.; Coste, S. C.; Graham, M. J.; Rogers, D. M.; Freedman, D. E. Employing Forbidden Transitions as Qubits in a Nuclear Spin-Free Chromium Complex. J. Am. Chem. Soc. 2016, 138, 1344-1348.

(19) Gatteschi, D.; Sessoli, R.; Villain, J. Molecular Nanomagnets (Mesoscopic Physics and Nanotechnology); Oxford University Press, USA, 2011.

(20) Gregson, M.; Chilton, N. F.; Ariciu, A.-M.; Tuna, F.; Crowe, I. F.; Lewis, W.; Blake, A. J.; Collison, D.; McInnes, E. J. L.; Winpenny, R. E. P.; Liddle, S. T. A Monometallic Lanthanide Bis(methanediide) Single Molecule Magnet with a Large Energy Barrier and Complex Spin Relaxation Behaviour. Chem. Sci. 2016, 7, 155-165.

(21) Blagg, R. J.; Ungur, L.; Tuna, F.; Speak, J.; Comar, P.; Collison, D.; Wernsdorfer, W.; McInnes, E. J. L.; Chibotaru, L. F.; Winpenny, R. E. P. Magnetic Relaxation Pathways in Lanthanide Single-Molecule Magnets. Nature Chem. 2013, 5, 673-678.

(22) Pedersen, K. S.; Dreiser, J.; Weihe, H.; Sibille, R.; Johannesen, H. V.; Sørensen, M. A.; Nielsen, B. E.; Sigrist, M.; Mutka, H.; Rols, S.; Bendix, J.; Piligkos, S. Design of SingleMolecule Magnets: Insufficiency of the Anisotropy Barrier as the Sole Criterion. Inorg. Chem. 2015, 54, 7600-7606. 
(23) Flanagan, B. M.; Bernhardt, P. V; Krausz, E. R.; Lu, S. R.; Riley, M. J. A Ligand-Field Analysis of the Trensal ( $\mathrm{H}_{3}$ Trensal) An Application of the Angular Overlap Model to Lanthanides. Inorg. Chem. 2002, 41, 5024-5033.

(24) Ishikawa, N.; Sugita, M.; Okubo, T.; Tanaka, N.; Iino, T.; Kaizu, Y. Determination of Ligand-Field Parameters and f-Electronic Structures of Double-Decker Bis(phthalocyaninato)lanthanide Complexes. Inorg. Chem. 2003, 42, 2440-2446.

(25) Ghosh, S.; Datta, S.; Friend, L.; Cardona-Serra, S.; Gaita-Ariño, A.; Coronado, E.; Hill, S. Multi-Frequency EPR Studies of a Mononuclear Holmium Single-Molecule Magnet Based on the Polyoxometalate $\left[\mathrm{Ho}^{\mathrm{III}}\left(\mathrm{W}_{5} \mathrm{O}_{18}\right)_{2}\right]^{9-}$. Dalton Trans. 2012, 41, 13697-13704.

(26) Perfetti, M.; Lucaccini, E.; Sorace, L.; Costes, J. P.; Sessoli, R. Determination of Magnetic Anisotropy in the LnTRENSAL Complexes ( $\mathrm{Ln}=\mathrm{Tb}$, Dy, Er) by Torque Magnetometry. Inorg. Chem. 2015, 54, 3090-3092.

(27) (a) Basler, R.; Boskovic, C.; Chaboussant, G.; Güdel, H. U.; Murrie, M.; Ochsenbein, S. T.; Sieber, A. Molecular Spin Clusters: New Synthetic Approaches and Neutron Scattering Studies. Chemphyschem 2003, 4, 910-926. (b) Baker, M. L.; Guidi, T.; Carretta, S.; Ollivier, J.; Mutka, H.; Güdel, H. U.; Timco, G. A.; McInnes, E. J. L.; Amoretti, G.; Winpenny, R. E. P.; Santini, P. Spin Dynamics of Molecular Nanomagnets Unravelled at Atomic Scale by Four-Dimensional Inelastic Neutron Scattering. Nat. Phys. 2012, 8, 906-911. (c) Furrer, A.; Waldmann, O. Magnetic Cluster Excitations. Rev. Mod. Phys. 2013, 85, 367-420.

(28) Kofu, M.; Yamamuro, O.; Kajiwara, T.; Yoshimura, Y.; Nakano, M.; Nakajima, K.; Ohira-Kawamura, S.; Kikuchi, T.; Inamura, Y. Hyperfine Structure of Magnetic Excitations in a Tb-Based Single-Molecule Magnet Studied by High-Resolution Neutron 
Spectroscopy. Phys. Rev. B 2013, 88, 064405.

(29) Marx, R.; Moro, F.; Dörfel, M.; Ungur, L.; Waters, M.; Jiang, S. D.; Orlita, M.; Taylor, J.;

Frey, W.; Chibotaru, L. F.; van Slageren, J. Spectroscopic Determination of Crystal Field Splittings in Lanthanide Double Deckers. Chem. Sci. 2014, 5, 3287-3293.

(30) Kettles, F. J.; Milway, V. A.; Tuna, F.; Valiente, R.; Thomas, L. H.; Wernsdorfer, W.; Ochsenbein, S. T.; Murrie, M. Exchange Interactions at the Origin of Slow Relaxation of the Magnetization in $\left\{\mathrm{TbCu}_{3}\right\}$ and $\left\{\mathrm{DyCu}_{3}\right\}$ Single-Molecule Magnets. Inorg. Chem 2014, 53, 8970-8978.

(31) Baker, M. L.; Tanaka, T.; Murakami, R.; Ohira-Kawamura, S.; Nakajima, K.; Ishida, T.; Nojiri, H. Relationship between Torsion and Anisotropic Exchange Coupling in a Tb ${ }^{\mathrm{III}}$ Radical-Based Single-Molecule Magnet. Inorg. Chem. 2015, 54, 5732-5738.

(32) Vonci, M.; Giansiracusa, M. J.; Gable, R. W.; Heuvel, W. Van Den; Latham, K.; Moubaraki, B.; Murray, K. S.; Yu, D.; Mole, R. A.; Soncini, A.; Boskovic, C. Ab Initio Calculations as a Quantitative Tool in the Inelastic Neutron Scattering Study of a SingleMolecule Magnet Analogue. Chem. Commun. 2016, 52, 2091-2094.

(33) Giansiracusa, M. J.; Vonci, M.; Van den Heuvel, W.; Gable, R. W.; Moubaraki, B.; Murray, K. S.; Yu, D.; Mole, R. A.; Soncini, A.; Boskovic, C. Carbonate-Bridged Lanthanoid Triangles: Single-Molecule Magnet Behavior, Inelastic Neutron Scattering, and $\mathrm{Ab}$ Initio Studies. Inorg. Chem. 2016, 55, 5201-5214.

(34) Pedersen, K. S.; Ungur, L.; Sigrist, M.; Sundt, A.; Schau-Magnussen, M.; Vieru, V.; Mutka, H.; Rols, S.; Weihe, H.; Waldmann, O.; Chibotaru, L. F.; Bendix, J.; Dreiser, J. Modifying the Properties of $4 \mathrm{f}$ Single-Ion Magnets by Peripheral Ligand Functionalisation. Chem. Sci. 2014, 5, 1650-1660. 
(35) Chilton, N. F.; Collison, D.; McInnes, E. J. L.; Winpenny, R. E. P.; Soncini, A. An Electrostatic Model for the Determination of Magnetic Anisotropy in Dysprosium Complexes. Nature Commun. 2013, 4, 2551.

(36) Clemente-Juan, J. M.; Coronado, E.; Gaita-Ariño, A. Mononuclear Lanthanide Complexes: Use of the Crystal Field Theory to Design Single-Ion Magnets and Spin Qubits. In Lanthanides and Actinides in Molecular Magnetism; Layfield, R. A., Murugesu, M., Eds.; Wiley-VCH Verlag \& Co. KGaA: Boschstr. 12, 69469 Weinheim, Germany, 2015; pp 27-59.

(37) Rechkemmer, Y.; Fischer, J. E.; Marx, R.; Dörfel, M.; Neugebauer, P.; Horvath, S.; Gysler, M.; Brock-Nannestad, T.; Frey, W.; Reid, M. F.; van Slageren, J. Comprehensive Spectroscopic Determination of the Crystal Field Splitting in an Erbium Single-Ion Magnet. J. Am. Chem. Soc. 2015, 137, 13114-13120.

(38) Baldoví, J. J.; Cardona-Serra, S.; Clemente-Juan, J. M.; Coronado, E.; Gaita-Ariño, A.; Palii, A. SIMPRE: A Software Package to Calculate Crystal Field Parameters, Energy Levels, and Magnetic Properties on Mononuclear Lanthanoid Complexes Based on Charge Distributions. J. Comput. Chem. 2013, 34, 1961-1967.

(39) Roos, B. O.; Taylor, P. R.; Sigbahn, P. E. M. A Complete Active Space SCF Method (CASSCF) Using a Density Matrix Formulated Super-CI Approach. Chemical Physics. 1980, pp 157-173.

(40) Roos, B. O.; Malmqvist, P.-Å. Relativistic Quantum Chemistry: The Multiconfigurational Approach; 2004; Vol. 6, p 2919.

(41) Chibotaru, L. F.; Ungur, L. Ab Initio Calculation of Anisotropic Magnetic Properties of Complexes. I. Unique Definition of Pseudospin Hamiltonians and Their Derivation. J. 
Chem. Phys. 2012, 137, 064112.

(42) Baldoví, J. J.; Clemente-Juan, J. M.; Coronado, E.; Duan, Y.; Gaita-Ariño, A.; GiménezSaiz, C. Construction of a General Library for the Rational Design of Nanomagnets and Spin Qubits Based on Mononuclear f-Block Complexes. The Polyoxometalate Case. Inorg. Chem. 2014, 53, 9976-9980.

(43) Qian, K.; Baldoví, J. J.; Jiang, S.-D.; Gaita-Ariño, A.; Zhang, Y.-Q.; Overgaard, J.; Wang, B.-W.; Coronado, E.; Gao, S. Does the Thermal Evolution of Molecular Structures Critically Affect the Magnetic Anisotropy? Chem. Sci. 2015, 6, 4587-4593.

(44) van Leusen, J.; Speldrich, M.; Schilder, H.; Kögerler, P. Comprehensive Insight into Molecular Magnetism via CONDON: Full vs. Effective Models. Coord. Chem. Rev. 2014, 289-290, 137-148.

(45) Clemente-Juan, J. M.; Coronado, E.; Gaita-Ariño, A. Magnetic Polyoxometalates: From Molecular Magnetism to Molecular Spintronics and Quantum Computing. Chem. Soc. Rev. 2012, 41, 7464-7478.

(46) Vonci, M.; Boskovic, C. Polyoxometalate-Supported Lanthanoid Single-Molecule Magnets. Aust. J. Chem. 2014, 67, 1542-1552.

(47) Suzuki, K.; Sato, R.; Mizuno, N.; Science, N. C. Reversible Switching of Single-Molecule Magnet Behaviors by Transformation of Dinuclear Dysprosium Cores in Polyoxometalates. Chem. Sci. 2013, 4, 596-600.

(48) Baldoví, J. J.; Cardona-Serra, S.; Clemente-Juan, J. M.; Coronado, E.; Gaita-Ariño, A.; Prima-García, H. Coherent Manipulation of Spin Qubits Based on Polyoxometalates: The Case of the Single Ion Magnet $\left[\mathrm{GdW}_{30} \mathrm{P}_{5} \mathrm{O}_{110}\right]^{14-}$. Chem. Commun. 2013, 49, 8922-8924.

(49) Shiddiq, M.; Komijani, D.; Duan, Y.; Gaita-Ariño, A.; Coronado, E.; Hill, S. Enhancing 
Coherence in Molecular Spin Qubits via Atomic Clock Transitions. Nature 2016, 531, 348-351.

(50) Peacock, R. D.; Weakley, T. J. R. Heteropolytungstate Complexes of the Lanthanide Elements. Part I. Preparation and Reactions. J. Chem. Soc. A 1971, 1836-1839.

(51) Sugeta, M.; Yamase, T. Crystal Structure and Luminescence Site of $\mathrm{Na}_{9}\left(\mathrm{EuW}_{10} \mathrm{O}_{36}\right) \cdot 32 \mathrm{H}_{2} \mathrm{O}$. Bull. Chem. Soc. Jpn. 1993, 66, 444-449.

(52) Roy, L. E.; Ortiz-Acosta, D.; Batista, E. R.; Scott, B. L.; Blair, M. W.; May, I.; Del Sesto, R. E.; Martin, R. L. Luminescence in $\mathrm{Ce}(\mathrm{IV})$ Polyoxometalate $\left[\mathrm{Ce}\left(\mathrm{W}_{5} \mathrm{O}_{18}\right)_{2}\right]^{8-}$ : A Combined Experimental and Theoretical Study. Chem. Commun. 2010, 46, 1848-1850.

(53) AlDamen, M. A.; Clemente-Juan, J. M.; Coronado, E.; Martí-Gastaldo, C.; Gaita-Ariño, A. Mononuclear Lanthanide Single-Molecule Magnets Based on Polyoxometalates. J. Am. Chem. Soc. 2008, 130, 8874-8875.

(54) AlDamen, M. A.; Cardona-Serra, S.; Clemente-Juan, J. M.; Coronado, E.; Gaita-Ariño, A.; Martí-Gastaldo, C.; Luis, F.; Montero, O. Mononuclear Lanthanide Single Molecule Magnets Based on the Polyoxometalates $\left[\mathrm{Ln}\left(\mathrm{W}_{5} \mathrm{O}_{18}\right)_{2}\right]^{9-}$ and $\left[\mathrm{Ln}\left(\beta_{2-}-\mathrm{SiW}_{11} \mathrm{O}_{39}\right)_{2}\right]^{13-}\left(\mathrm{Ln}^{\mathrm{III}}=\right.$ Tb, Dy, Ho, Er, Tm, and Yb). Inorg. Chem. 2009, 48, 3467-3479.

(55) Luis, F.; Martínez-Pérez, M. J.; Montero, O.; Coronado, E.; Cardona-Serra, S.; MartíGastaldo, C.; Clemente-Juan, J. M.; Sesé, J.; Drung, D.; Schurig, T. Spin-Lattice Relaxation via Quantum Tunneling in an $\mathrm{Er}^{3+}$-Polyoxometalate Molecular Magnet. Phys. Rev. B 2010, 82, 60403 .

Baldoví, J. J.; Cardona-Serra, S.; Clemente-Juan, J. M.; Coronado, E.; Gaita-Ariño, A.; Palii, A. Rational Design of Single-Ion Magnets and Spin Qubits Based on Mononuclear Lanthanoid Complexes. Inorg. Chem. 2012, 51, 12565-12574. 
(57) Martínez-Pérez, M. J.; Cardona-Serra, S.; Schlegel, C.; Moro, F.; Alonso, P. J.; PrimaGarcía, H.; Clemente-Juan, J. M.; Evangelisti, M.; Gaita-Ariño, A.; Sesé, J.; Van Slageren, J.; Coronado, E.; Luis, F. Gd-Based Single-Ion Magnets with Tunable Magnetic Anisotropy: Molecular Design of Spin Qubits. Phys. Rev. Lett. 2012, 108, 1-5.

Li, Y.-Y. Y.-Z.; Gao, F.; Beves, J. E.; Li, Y.-Y. Y.-Z.; Zuo, J.-L. A Giant MetalloSupramolecular Cage Encapsulating a Single-Molecule Magnet. Chem. Commun. 2013, $49,3658-3660$.

(59) Aravena, D.; Venegas-Yazigi, D.; Ruiz, E. Single-Molecule Magnet Properties of Transition-Metal Ions Encapsulated in Lacunary Polyoxometalates: A Theoretical Study. Inorg. Chem. 2016, doi: acs.inorgchem.6b00145.

(60) CrysAlis PRO v171.37.35. Agilent Technologies Ltd; Yarnton, Oxfordshire, England., 2014.

(61) Sheldrick, G. M. SHELXT - Integrated Space-Group and Crystal-Structure Determination. Acta Cryst. 2015, A71, 3-8.

(62) Sheldrick, G. M. Crystal Structure Refinement with SHELXL. Acta Cryst. 2015, C71, 38.

(63) Dolomanov, O. V.; Bourhis, L. J.; Gildea, R. J.; Howard, J. A K.; Puschmann, H. OLEX2: A Complete Structure Solution, Refinement and Analysis Program. J. Appl. Crystallogr. 2009, 42, 339-341.

(64) Yu, D.; Mole, R.; Noakes, T.; Kennedy, S.; Robinson, R. Pelican. A Time of Flight Cold Neutron Polarization Analysis Spectrometer at OPAL. J. Phys. Soc. Jpn. 2013, 82, SA027.

(65) Richard, D.; Ferrand, M.; Kearley, G. J. Analysis and Visualisation of Neutron-Scattering Data. J. Neutron Res. 1996, 4, 33-39. 
(66) Pintschovius, L.; Reznik, D.; Weber, F.; Bourges, P.; Parshall, D.; Mittal, R.; Chaplot, S. L.; Heid, R.; Wolf, T.; Lamago, D.; Lynn, J. W. Spurious Peaks Arising from Multiple Scattering Events Involving the Sample Environment in Inelastic Neutron Scattering. $J$. Appl. Crystallogr. 2014, 47, 1472-1476.

(67) Rotter, M.; Boothroyd, A. T. Going beyond the Dipole Approximation to Improve the Refinement of Magnetic Structures by Neutron Diffraction. Phys. Rev. B 2009, 79, 140405 .

(68) Rudowicz, C. Transformation Relations for the Conventional $\mathrm{O}_{\mathrm{k}}{ }^{\mathrm{q}}$ and Normalised $\mathrm{O}_{\mathrm{k}}{ }^{\mathrm{q}}$ Stevens Operator Equivalents with $\mathrm{k}=1$ to 6 and $-\mathrm{k} \leq \mathrm{q} \leq \mathrm{k}$. J. Phys. C Solid State Phys. 1985, $18,1415-1430$.

(69) Görller-Walrand, C.; Binnemans, K. Rationalization of Crystal-Field Parametrization. In Handbook on the Physics and Chemistry of Rare Earths Volume 23; 1996; pp 121-283.

(70) Kirkpatrick, S.; Gelatt, C. D.; Vecchi, M. P. Optimization by Simulated Annealing. Science 1983, 220, 671-680.

(71) Stoll, S.; Schweiger, A. EasySpin, a Comprehensive Software Package for Spectral Simulation and Analysis in EPR. J. Magn. Reson. 2006, 178, 42-55.

(72) Aquilante, F.; De Vico, L.; Ferré, N.; Ghigo, G.; Malmqvist, P.-A.; Neogrády, P.; Pedersen, T. B.; Pitonák, M.; Reiher, M.; Roos, B. O.; Serrano-Andrés, L.; Urban, M.; Veryazov, V.; Lindh, R. MOLCAS 7: The next Generation. J. Comput. Chem. 2010, 31, 224-247.

(73) Veryazov, V.; Widmark, P.-O.; Serrano-Andres, L.; Lindh, R.; Roos, B. O. 2MOLCAS as a Development Platform for Quantum Chemistry Software. Int. J. Quantum Chem. 2004, $100,626-635$. 
(74) Karlström, G.; Lindh, R.; Malmqvist, P.-Å.; Roos, B. O.; Ryde, U.; Veryazov, V.; Widmark, P.-O.; Cossi, M.; Schimmelpfennig, B.; Neogrady, P.; Seijo, L. MOLCAS: A Program Package for Computational Chemistry. Comput. Mater. Sci. 2003, 28, 222-239.

(75) Wahlgren, U. The Effective Core Potential Method. In Lecture Notes in Quantum Chemistry; Roos, B. O., Ed.; Lecture Notes in Chemistry; Springer Berlin Heidelberg: Berlin, Heidelberg, 1992; Vol. 58, pp 413-421.

(76) Seijo, L.; Barandiarán, Z. The Ab Initio Model Potential Method: A Common Strategy for Effective Core Potential and Embedded Cluster Calculations. In Computational Chemistry: Reviews of Current Trends; Leszczynski, J., Ed.; World Scientific: Singapur, $1999 ;$ p 55.

(77) Ungur, L.; Chibotaru, L. F. Computational Modelling of the Magnetic Properties of Lanthanide Compounds. In Lanthanides and Actinides in Molecular Magnetism; WileyVCH Verlag GmbH \& Co. KGaA: Weinheim, Germany, 2015; pp 153-184.

(78) Yamase, T.; Ozeki, T.; Tosaka, M. Octasodium Hydrogen Decatungstogadolinate Triacontahydrate. Acta Cryst. 1994, C50, 1849-1852.

(79) Sawada, K.; Yamase, T. Nonasodium Decatungstodysprosate Pentatriacontahydrate. Acta Cryst. 2002, C58, i149-i151.

(80) Barsukova, M.; Dickman, M. H.; Visser, E.; Sankar Mal, S.; Kortz, U. Synthesis and Structural Characterization of the Yttrium Containing Isopolytungstate $\left[\mathrm{YW}_{10} \mathrm{O}_{36}\right]^{9-} . \mathrm{Z}$. Anorg. Allg. Chem. 2008, 634, 2423-2427.

(81) Casanova, D.; Llunell, M.; Alemany, P.; Alvarez, S.; D. Casanova, M Llunell, P.; Alemany, S. A. The Rich Stereochemistry of Eight-Vertex Polyhedra: A Continuous Shape Measures Study. Chem. Eur. J. 2005, 11, 1479-1494. 
(82) Llunell, M.; Casanova, D.; Cirera, J.; Bofill, J. M.; Alemany, P.; Alvarez, S.; Pinsky, M.; Avnir, D. SHAPE 2.0; Universitat de Barcelona and The Hebrew University of Jerusalem: Barcelona, 2003.

(83) Das, C.; Upadhyay, A.; Vaidya, S.; Singh, S. K.; Rajaraman, G.; Shanmugam, M. Origin of SMM Behaviour in an Asymmetric Er(III) Schiff Base Complex: A Combined Experimental and Theoretical Study. Chem. Commun. 2015, 51, 6137-6140.

(84) Kerridge, A. The Complete-Active-Space Self-Consistent-Field Approach and Its Application to Molecular Complexes of the F-Elements. In Computational Methods in Lanthanide and Actinide Chemistry; John Wiley \& Sons Ltd: Chichester, UK, 2015; pp $121-146$.

(85) Furrer, A.; Güdel, H. Interference Effects in Neutron Scattering from Magnetic Clusters. Phys. Rev. Lett. 1977, 39, 657-660.

(86) Baker, J. M. EPR and ENDOR in the Lanthanides. In Electron Spin Resonance; Symons, M. C. R., Ed.; Electron Spin Resonance; Royal Society of Chemistry: Cambridge, 1993; Vol. 13, pp 131-177.

(87) Sears, V. F. Neutron Scattering Lengths and Cross Sections. Neutron News 1992, 3, $26-$ 37.

(88) Cucinotta, G.; Perfetti, M.; Luzon, J.; Etienne, M.; Car, P.-E.; Caneschi, A.; Calvez, G.; Bernot, K.; Sessoli, R. Magnetic Anisotropy in a Dysprosium/DOTA Single-Molecule Magnet: Beyond Simple Magneto-Structural Correlations. Angew. Chemie Int. Ed. 2012, $51,1606-1610$.

(89) Boulon, M.-E.; Cucinotta, G.; Luzon, J.; Degl'Innocenti, C.; Perfetti, M.; Bernot, K.; Calvez, G.; Caneschi, A.; Sessoli, R. Magnetic Anisotropy and Spin-Parity Effect along 
the Series of Lanthanide Complexes with DOTA. Angew. Chem. Int. Ed. 2013, 52, 350354. 


\section{Table of Contents Graphic and Synopsis}

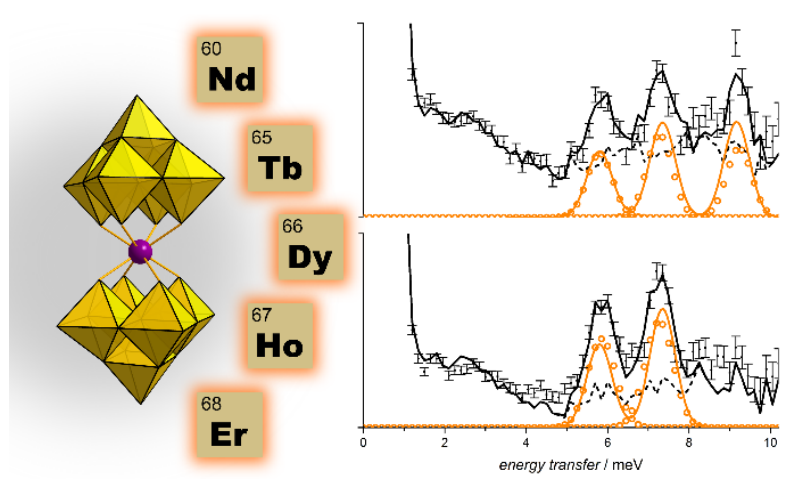

Inelastic neutron scattering (INS) and $a b$ initio Complete Active Space Self Consistent Field/Restricted Active Space State Interaction calculations have been used to investigate the crystal field (CF) splitting of analogs of the $\mathrm{Na}_{9}\left[\mathrm{Ln}\left(\mathrm{W}_{5} \mathrm{O}_{18}\right)_{2}\right]$ single-molecule magnet (SMM) family. Magnetic CF excitations were observed for all compounds and ab initio calculations supported the assignment of the allowed INS transitions, allowing very good reproduction of the energy levels and magnetic properties. Ultimately we have established the relationship between the wavefunction composition of the $\mathrm{CF}$ split $\mathrm{Ln}^{\mathrm{III}}$ ground multiplets and the experimentally measured magnetic and spectroscopic properties, improving at the same time the current state-ofthe-art theoretical models for this family of SMMs. 